\title{
m6A modifications regulate intestinal immunity and rotavirus infection
}

Anmin Wang ${ }^{1,2}$, Wanyin Tao ${ }^{1,2}$, Jiyu Tong ${ }^{3}$, Juanzi Gao², Jinghao Wang2, Gaopeng $\mathrm{Hou}^{4}$, Cheng Qian${ }^{2}$, Guorong Zhang ${ }^{1}$, Runzhi Li ${ }^{1}$, Decai Wang${ }^{1}$, Xingxing Ren ${ }^{1}$, Kaiguang Zhang'2, Siyuan Ding" , , Wen Pan ${ }^{1,2 \#, ~ H u a-B i n g ~ L i 3, \#, ~ R i c h a r d ~ F l a v e l l 5,6, \#, ~}$ Shu Zhu',2,7,8,\#

\section{Affiliations}

${ }^{1}$ Hefei National Laboratory for Physical Sciences at Microscale, the Chinese Academy of Sciences Key Laboratory of Innate Immunity and Chronic Disease, School of Basic Medical Sciences, Division of Life Sciences and Medicine, University of Science and Technology of China, 230027 Hefei, China.

${ }^{2}$ Department of Digestive Disease, The First Affiliated Hospital of University of Science and Technology of China, Division of Life Sciences and Medicine, University of Science and Technology of China, 230001 Hefei, China.

${ }^{3}$ Shanghai Institute of Immunology, Department of Microbiology and Immunology, Shanghai Jiao Tong University School of Medicine (SJTU-SM), Shanghai 200025, China.

${ }^{4}$ Department of Molecular Microbiology, Washington University School of Medicine in St. Louis, St. Louis, Missouri, USA.

${ }^{5}$ Department of Immunobiology, Yale University School of Medicine, 300 Cedar Street, New Haven, CT 06510, USA.

${ }^{6}$ Howard Hughes Medical Institute, Yale University School of Medicine, 300 Cedar Street, New Haven, CT 06510, USA.

${ }^{7}$ School of Data Science, University of Science and Technology of China, 230026 Hefei, China.

${ }^{8} \mathrm{CAS}$ Centre for Excellence in Cell and Molecular Biology, University of Science and Technology of China, Hefei, China.

\#Correspondence should be addressed to Shu Zhu (zhushu@ustc.edu.cn) and HuaBing Li (huabing.li@shsmu.edu.cn) 


\section{Abstract}

N6-methyladenosine (m6A) is an abundant mRNA modification and affects many biological processes. However, how m6A levels are regulated during physiological or pathological processes such as virus infections, and the in vivo function of m6A in the intestinal immune defense against virus infections are largely unknown. Here, we uncover a novel antiviral function of m6A modification during rotavirus (RV) infection in small bowel intestinal epithelial cells (IECs). We found that rotavirus infection induced global m6A modifications on mRNA transcripts by down-regulating the m6a eraser ALKBH5. Mice lacking the $\mathrm{m}^{6} \mathrm{~A}$ writer enzymes METTL3 in IECs (Mettl3 ${ }^{\mathrm{AIEC}}$ ) were resistant to RV infection and showed increased expression of interferons (IFNs) and IFN-stimulated genes (ISGs). Using RNAsequencing and m6A RNA immuno-precipitation (RIP)-sequencing, we identified IRF7, a master regulator of IFN responses, as one of the primary m6A targets during virus infection. In the absence of METTL3, IECs showed increased Irf7 mRNA stability and enhanced type I and III IFN expression. Deficiency in IRF7 attenuated the elevated expression of IFNs and ISGs and restored susceptibility to RV infection in Mettl3 ${ }^{\Delta \mathrm{IEC}}$ mice. Moreover, the global m6A modification on mRNA transcripts declined with age in mice, with a significant drop from 2 weeks to 3 weeks post birth, which likely has broad implications for the development of intestinal immune system against enteric viruses early in life. Collectively, we demonstrated a novel host m6A-IRF7-IFN antiviral signaling cascade that restricts rotavirus infection in vivo.

\section{Keywords}

Rotavirus, N6-methyladenosine, METTL3, ALKBH5, IFNs

\section{Introduction}

N6-methyladenosine (m6A) is the most abundant internal mRNA modification and modulates diverse cellular functions through m6A-related writers, erasers, and 
readers[1-3]. The m6A modification directly recruits m6A-specific proteins of the YT521-B homology (YTH) domain family[1]. These proteins mediate the m6Adependent regulation of pre-mRNA processing, microRNA processing, translation initiation, and mRNA decay[1]. In recent works, m6A modifications has been identified in the genomes of RNA viruses and the transcripts of DNA viruses with either a pro-viral or anti-viral role[4-8]. Furthermore, m6A RNA modificationmediated down-regulation of the $\alpha$-ketoglutarate dehydrogenase (KGDH)itaconate pathway inhibits viral replication independent of the innate immune response[9]. According to Gao et al. (2020), m6A modification preserves the selfrecognition of endogenous transcripts. Deletion of the m6A writer Mettl3 decreases the m6A modifications in endogenous retrovirus (ERV) transcripts. The accumulation of ERVs activates pattern recognition receptors (e.g. RIG-I) pathways, resulting in a detrimental interferon response in livers of fetal mice[10].

The m6A modification of the enterovirus 71 (EV71) RNA genome is important for viral propagation, and EV71 infection increases the expression of m6A writers in vitro[11]. m6A methyltransferase Mettl3 knockdown reduces whereas m6A demethylase FTO knockdown increases EV71 replication[11]. In addition, human cytomegalovirus can up-regulate the expression of m6A-related proteins[12, 13]. Despite the knowledges about m6A regulation and function during viral infection revealed by these in vitro studies, the regulation of m6A modifications and the specific role of $\mathrm{m} 6 \mathrm{~A}$ in the anti-viral response in vivo, especially in the gastrointestinal tract, remains unclear.

Rotavirus (RV), a member of the family Reoviridae, is a nonenveloped icosahedralstructured virus with 11 segments of double-stranded RNA. Children under the age of five are at high risk of rotavirus infection, which causes severe diarrhea, dehydration, and death[14]. Rotaviruses encode multiple viral proteins to inhibit innate immune responses by degrading interferon regulatory factors (IRFs) and mitochondrial antiviral-signaling protein (MAVS), thus facilitating efficient virus infection and replication $[15,16]$. The timely induction of an IFN response is key to the host successful control of invading viruses, including RV[17-19]. Here, we 
found rotavirus infection induced global m6A modifications on mRNA transcripts by down-regulating the $\mathrm{m} 6 \mathrm{a}$ eraser ALKBH5. Mice lacking the $\mathrm{m}^{6} \mathrm{~A}$ writer enzymes METTL3 in IECs (Mettl3 ${ }^{\Delta \mathrm{IEC}}$ ) were resistant to RV infection. We identified IRF7, a master regulator of IFN responses[17], as one of the primary m6A targets during virus infection. In the absence of METTL3, IECs showed increased Irf7 mRNA stability and enhanced type I and III IFN expression. Deficiency in IRF7 attenuated the elevated expression of IFNs and ISGs and restored susceptibility to RV infection in Mettl $3^{\triangle \mathrm{IEC}}$ mice. Collectively, we identified a novel regulation and function of m6A modifications in an enteric viral infection model in vivo.

\section{Results}

\section{The regulation and function of mRNA m6A modifications during rotavirus} infection

Rotavirus infections primarily take place in children under the age of five in humans and in neonatal mice younger than 2 weeks-old[14, 20]. Intriguingly, total RNA m6A modifications in the mouse ileal tissues, revealed by a m6A dot blot, significantly declined from 2 weeks to 3 weeks post birth (Fig. 1a and 1b), which caused by increased Alkbh5 expression (Fig. 1c). Besides, the global m6A RNA modification levels increased in the ileum tissue of suckling mice post RV murine strain EW infection (Fig. 1d and 1e). As a control, the global m6A levels decreased in Mettl3 depleted bone marrow derived macrophages (BMDMs) (Fig. 1d). Thus, we hypothesize that RV may induce an enriched cellular m6A modification environment and a weakened innate immune response to facilitate virus replication. To investigate the in vivo role of m6A in the anti-RV immunity, we conditionally knocked out the m6A writer Mettl3 in IECs (Mettl3 f/f vil-cre, $M e t t 3^{\Delta \mathrm{IEC}}$ ), which is specifically infected by RV. Following infection with RV EW strain, the viral RNA load in Mettl3 ${ }^{\mathrm{AIC}}$ mice ileum tissue was significantly lower than that in the wild-type (WT) littermates (Fig. 1f and s1). Fecal virus shedding was also significantly lower in Mettl3 ${ }^{\Delta \mathrm{IEC}}$ mice (Fig. 1g). Genetic knockdown of METTL3 in HT-29 cells, a human colonic epithelial cell line, by CRISPR-mediated 
gene silencing, also led to reduced RV replication (Fig. 1h), further highlighting the resistance phenotype to RV infection by METTL3 deficiency.

\section{Mettl3 deficiency in IECs results in decreased m6A deposition on $\operatorname{Irf} 7$, and}

\section{increased interferon responses}

To dissect the underlying mechanism, we performed RNA-sequencing using the IECs from Mettl3-deficient mice and littermate controls at steady state. Most of the differentially expressed genes in Mettl3-deficient IECs vs WT IECs were enriched in the pathways of "defense response to virus", "response to interferon-beta", and "positive regulation of innate immune response" by gene ontology analysis (Fig. 2a). Heatmap also showed that a panel of interferon stimulate genes (ISGs) are upregulated in Mettl3-deficient IECs compared to WT IECs (Fig. 2b). To map potential m6A modification sites on mRNAs of these differential expressed genes in IECs, we conducted m6A RIP-sequencing based on a previously reported protocol [5]. We found that m6A modified one of the master regulators of IFNs, Irf7 (Fig.2c), which played a key role in the network of these differential expressed genes in Mettl3-deficient IECs compared to WT IECs, analyzed by STRING (Fig.2d). Of note, IRF7 was the only IRFs that highly expressed in Mettl3-deficient IECs, and IRF7 was the prominently highest expressed IRFs in IECs (Fig. 2e), indicating IRF7 might be one of the key genes that are modulated by m6A modifications. In addition, the m6A peak was primarily located on the 5' UTR and 3' UTR of Irf7 in ileum IECs (Fig. 2d). We also validated our results by m6A RIP-qPCR to examine m6A modification sites in Irf7 mRNA based on our RIP-sequencing data and predicted results from the database (http://rna.sysy.edu.cn) (Fig. s2a and s2b). It should be noted that our m6A-RIP-seq did not identified previous reported IFN[12, 13], possibly due to the low expression level of IFN $b$ in IECs at steady state.

IRF7 is a known master regulator of Type I interferon and Type III interferondependent immune responses in respond to virus infection[16, 17, 21]. We reasoned that loss of m6A modification on Irf7 mRNA is responsible for the 
153 increased IFN response and subsequent resistance to RV infection. Thus, we first validated the regulation of $\operatorname{Irf} 7 \mathrm{mRNA}$ levels by m6A in mice and in cells. We found an increase of $\operatorname{Irf} 7 \mathrm{mRNA}$ in ileum tissue of $M e t t l 3^{\Delta \mathrm{IEC}}$ mice compared to that in WT littermate control mice (Fig. 2f). Consistently, the expression of Irf7 mRNA was also higher in METTL3 knockdown HT-29 and METTL3 knockout rhesus monkey MA104 cells, suggesting that the regulation of Irf7 expression by m6A is likely conserved across species (Fig. s3a, s3b, s4a, and s4b). Furthermore, genetic knockdown of METTL3 in HT-29 also led to increased IFN responses (Fig. s3). As $\mathrm{m} 6 \mathrm{~A}$ is known to regulate the mRNA decay, we next sought to determine whether the stability of Irf7 mRNA is regulated by m6A. We used actinomycin D to block the de novo RNA synthesis in HT-29 cells to assess the RNA degradation by METTL3 knockdown. The Irf7 mRNA degraded significantly slower in Mettl3-knockdown HT-29 cells than the control cell line (Fig. 2g, s3b).

To directly evaluate the role of m6A in modulating the stability of $\operatorname{Irf7}$ mRNA, luciferase reporter assays were conducted. In comparison with wild-type Irf73'UTR (Irf7-WT) constructs, the ectopically expressed constructs harboring m6A mutant Irf7-3'UTR (Irf7-MUT) showed significantly increased luciferase activity (Fig. 2h). These results suggest that the upregulation of $\operatorname{Irf} 7$ mRNA level in Mettl3 ${ }^{\Delta \mathrm{IEC}}$ mice is caused by the loss of m6A modification mediated mRNA decay. To evaluate the potential influence of m6A on IRF7 transcriptional targets, we also measured the expressions of IFNs and ISGs in rotavirus infected ileum tissue from Mettl3 ${ }^{\mathrm{IIEC}}$ mice and littermate WT mice. We found the transcriptional targets of Irf7, were all up-regulated in Mettl3 ${ }^{\mathrm{DIEC}}$ mice (Fig. 2i). Furthermore, we found that the mRNAs of Irf7 and its transcriptional targets ISGs increased in the ileum of the mice from 1 to 4 weeks, with a dramatic up-regulation from 2 to 3 weeks (Fig. s5), which was concomitant with the decrease of global m6A modifications (Fig. 1a). These results demonstrated that METTL3 deficiency in IECs results in decreased m6A deposition on IRF7, and increased interferon response. 
IRF7 Deficiency attenuated the increased interferon response and resistance to rotavirus infection in $\mathrm{Mettl}^{\mathrm{\Delta IEC}}$ mice

185 To determine whether Irf7 plays a key role in the resistance phenotype to RV infection in Mettl3 deficient mice in IECs, we crossed $\operatorname{Irf} 7^{-/-}$mice to Mettl3 ${ }^{\Delta \mathrm{IEC}}$ mice. Following RV oral gavage, the expression of IFNs and ISGs in ileum from $\operatorname{Irf7-/-~}$ Mettl3 $3^{\Delta \mathrm{IEC}}$ mice were significantly lower than those from Mettl3 $3^{\Delta \mathrm{IEC}}$ mice at $2 \mathrm{dpi}$ (Fig 3a-c), and unlike the increased expression of IFNs and ISGs in Mettl3 ${ }^{\Delta I E C}$ mice vs littermate WT controls, by deficiency of IRF7, the expression of IFNs and ISGs in ileum from $\operatorname{Irf7}^{-/-}$Mettl3 ${ }^{\mathrm{IEC}}$ mice were not significantly different from those from Irf7-/- mice (Fig 3a-c), suggesting that Irf7 mediates the increased expression of IFNs and ISGs in Mettl3 ${ }^{\mathrm{IIEC}}$ mice.

Moreover, the $\operatorname{Irf} 7^{-/-}$Mettl${ }^{\triangle \mathrm{IEC}}$ mice showed significantly higher viral loads in ileum tissue and higher fecal shedding of RV Mettl3 ${ }^{\Delta I E C}$ (Fig. 3d-f). Similarly, unlike the much lower fecal viral shedding in Mettl3 ${ }^{\mathrm{IIEC}}$ mice vs littermate WT controls,

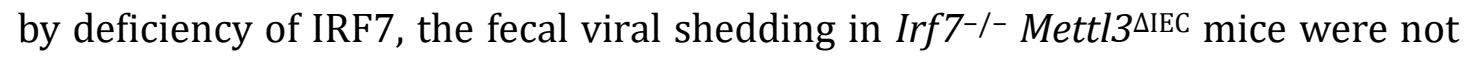
significantly different from those in $\operatorname{Irf} 7^{-/-}$mice (Fig 3d), suggesting that Irf7 mediates the resistant phenotype of rotavirus infection measured by fecal viral shedding in Mettl3 ${ }^{\Delta \mathrm{IEC}}$ mice. Notably, the viral proteins expression difference in ileum from $\operatorname{Irf7}^{-/-}$Mettl${ }^{\mathrm{AIEC}}$ mice vs that from $\operatorname{Irf7-/-}$ mice (9.7-fold lower for NSP2 and 9.3-fold lower for VP7), was much lower than the viral proteins expression difference in ileum from Mettl3 ${ }^{\Delta \mathrm{IEC}}$ mice vs that from littermate mice (267.1-fold lower for NSP2 and 283.4-fold lower for VP7) (Fig 3e), suggesting that besides the contribution of $\operatorname{Irf} 7$ to the resistant phenotype of rotavirus infection in IECs from Mettl3 ${ }^{\triangle \mathrm{IEC}}$ mice, other pathways (e.g. m6A modifications in RV RNA) may also play roles. Therefore, IRF7 is an important mediator of the increased IFNs and ISGs phenotype of Mettl3 ${ }^{\Delta \mathrm{IEC}}$ mice to RV infection. 


\section{Rotavirus suppresses ALKBH5 expression through NSP1 to evade immune} defense

We next sought to determine how RV regulates the m6A modifications in IECs. We first measured whether RV infection regulates the m6A-related writer and eraser proteins in the intestine. The protein levels of the methyltransferases METTL3 and METTL14 and demethylase FTO were not affected by RV infection in ileum tissue (Fig. $4 \mathrm{a}$ and b). In contrast, the protein level of demethylase ALKBH5 was significantly down-regulated by RV infection in the ileum (Fig. 4a, 4b). To determine whether ALKBH5 play a role in anti-RV infection since it's suppressed during RV infection, we generated the IEC-specific deletion of ALKBH5 in mice (Alkbh5 f/f vil-cre, Alkbh5 ${ }^{\Delta \mathrm{IEC}}$ ). The depletion of ALKBH5 in IECs did not affected the anti-RV immune response (Fig. 4c), the viral shedding in the feces (Fig.4d), or the viral protein expression in the ileum (Fig. 4e), likely due to the suppressed ALKBH5 expression in ileum tissue of WT mice infected by RV.

Non-structural protein 1 (NSP1) is a well-established RV-encoded innate immune antagonist that are showed to degrade IRF3 and $\beta$-Trcp $[22,23]$. To test the potential role of NSP1 in AlKBH5 inhibition, we used the recently developed reverse genetics system and rescued recombinant WT RV SA11 strain and a mutant virus that does not express NSP1 (NSP1-null)[24]. We infected HEK293 cells with WT and NSP1-null RVs, only WT RV reduced ALKBH5 protein levels (Fig. 4f), suggesting that the down-regulation of ALKBH5 expression by RV is NSP1dependent. These results suggest that RV might evades the anti-viral immune response via downregulation of ALKBH5 expression.

\section{Discussion}

Previous studies reported that m6A modifications on mRNA in mice embryonic fibroblasts or normal human dermal fibroblasts negatively regulate the IFN response by accelerating the mRNA degradation of type I IFNs [12, 13]. However, these studies were mainly conducted in vitro, leaving the relationship between 
m6A and IFN pathway in vivo an unexplored territory. Since type I and type III IFNs play a critical role in the antiviral immune response in the GI tract[19], we used an RV infection model, in which IECs are specifically infected, as well as conditional knockout mice with IECs-specific depletion of m6A writer METTL3 or m6A eraser ALKBH5, to study the role of m6A modification in regulating IFN response towards rotavirus in IECs. Using RNA-seq and m6A-seq techniques, we identified IRF7, a key transcription factor upstream of IFNs and ISGs, as one of the m6A modified targets during RV infection, and IRF7 is essential to mediate the elevated antirotavirus immune response by METTL3 deficiency. These results identify Irf7 as an important m6A target, and characterize, for the first time, the regulation of IFN response during RV infection in intestine, enabling a better understanding of how m6A modifications on mRNAs regulate anti-viral innate immune responses.

In addition to directly regulating target genes that involved in innate immune pathways, m6A modification is also known to affect viral gene expression, replication and generation of progeny virions $[1,25]$. Some viral RNA genomes are modified by m6A, such as simian virus 40[4, 26], influenza $A$ virus[27], adenovirus[28], avian sarcoma virus[29], Rous Sarcoma virus[30], hepatitis C virus[31], and Zika virus[32]. Through our m6A-RIP-qPCR, Rotavirus RNA was also found to have m6A modification (Figure s6). Of note, genetic deletion of METTL3 in monkey kidney MA104 cell line, which has limited IFN responses[33], also led to reduced RV replication. Further, IRF7 deficiency didn't fully restore the suppressed rotaviral infection in Mettl3 ${ }^{\Delta \mathrm{IEC}}$ mice (Fig 3e). These results suggest that besides the contribution of $\operatorname{Irf} 7$ to the resistant phenotype of rotavirus infection in IECs from Mettl3 ${ }^{\triangle I E C}$ mice, other pathways (e.g. m6A modifications in RV RNA) may also play roles. The detailed mechanisms warrant further investigations in the future. 
retrovirus (ERV) mRNA, or by regulating ERV heterochromatin and inhibiting its transcription[2, 3]. The absence of m6A results in the formation of abnormal endogenous dsRNA, which causes an aberrant immune response and necrosis in the hematopoietic system[10]. In the intestine, through immunostaining with J2 antibody, we detected the increase of dsRNA levels in IECs of Mettl3 ${ }^{\triangle I E C}$ mice compare to the littermate WT mice (Figure s7). In consistency, the aforementioned RNA-seq data showed that the expression of a set of ISGs including IRF7 was significantly upregulated in Mettl3 KO IECs. Our data suggest a dual activation model in steady state that in the absence of METTL3, the increase of dsRNA will induce the IFN responses, and the increase of mRNA stability of Irf7, which serves as a key transcription factor of IFN and ISG expression, will amplify this process. Furthermore, m6A modification of viral RNA genomes affect the activation of innate sensor-mediated signaling[34,35]. Decreased m6A modification on RV genomes may activate innate sensors directly and induce higher IFN response, which will also be amplified by increase of mRNA stability of Irf7.

Although m6A is involved in many important biological processes, the regulation of m6A modifications remain poorly understood. Here, we found RV infection down-regulates the level of m6A eraser ALKBH5 to induce m6A modifications, in an NSP1 dependent manner. The precise mechanism remains to be examined. As a result, $A L K B H 5$ deficiency in IECs results in normal susceptibility to RV infection. In addition, the global m6A modification on mRNA transcripts declines by ages in the intestine, with a significant drop from 2 to 3 weeks post birth, which implicate the drop of RV infectivity in adult mice vs neonatal mice. The dual regulation of m6A levels during RV infection and development provide new insights into the choice of either $\mathrm{RV}$ or the host on regulation of m6A modification to achieve either immune evasion or immune surveillance, respectively.

In conclusion, our work shed light into a novel role of m6A modifications in RV infection in vivo, and reported a tissue specific regulation of $\mathrm{m} 6 \mathrm{~A}$ during $\mathrm{RV}$ 
302

303

304

305

306

307

308

309

310

311

312

313

314

315

316

317

318

319

320

321

322

323

infection or development (Figure 4g). Future studies on tissue-specific regulation of m6A modification by viral infections in other tissues and organs (e.g. lung, liver) will be of interest.

\section{Materials and Methods}

Mice

Mettl3 conditional knockout mice were generated by inserting two loxp sites into the intron after the first exon and the intron before the last exon of Mettl3 using CRISPR/cas9 based genome-editing system as previously described[5]. Alkbh5 conditional knockout mice were generated by inserting two loxp sites into the introns flanking the first exon of Alkbh5 using CRISPR/cas9 based genome-editing system as previously described[36]. Genotyping of Mettl3f/f mice, Alkbh5f/f mice, Vil-cre mice (The Jackson Laboratory, Stock No: 021504), and Irf7\%/- mice (RIKEN BRC, RBRC01420) were confirmed by PCR using primers as list below:

Mettl3 f/f mice

Mettl3-L1+: CCCAACAGAGAAACGGTGAG

Mettl3-L2-: GGGTTCAACTGTCCAGCATC

Vil-cre mice

Vil-Cre-182/150-F: GCCTTCTCCTCTAGGCTCGT

Vil-Cre-182-R: TATAGGGCAGAGCTGGAGGA

Vil-Cre-150-R: AGGCAAATTTTGGTGTACGG

Irf7 $\%$ mice

RBRC01420-Irf7-WT-F : GTGGTACCCAGTCCTGCCCTCTTTATAATCT

RBRC01420-Irf7-Mut-F : TCGTGCTTTACGGTATCGCCGCTCCCGATTC

RBRC01420-Irf7-R: AGTAGATCCAAGCTCCCGGCTAAGTTCGTAC

Alkbh5 f/f mice

Alkbh5-L1+: GCACAGTGGAGCACATCATG

Alkbh5-L2-: CAGAGGGCAAGCAACCACAC

The sex-, age- and background-matched littermates of the knockout or conditional 
331 knockout mice were used as the controls in the present study. All mice were on the C57BL/6 background. Mice were maintained in SPF conditions under a strict $12 \mathrm{~h}$ light cycle (lights on at 08:00 and off at 20:00). All animal studies were performed according to approved protocols by the Ethics Committee at the University of Science and Technology of China (USTCACUC202101016).

\section{Cell culture}

The MA104 cell line was obtained from the Cell Resource Center, Peking Union Medical College (which is the headquarter of National Infrastructure of Cell Line Resource). The identity of the cell line was authenticated with STR profiling (FBI, CODIS). The results can be viewed on the website (http://cellresource.cn). HEK293T (ATCC CRL-3216), HT-29 (ATCC HTB-38D ${ }^{\mathrm{TM}}$ ) were obtained from the American Type Culture Collection (ATCC). All of these cells were cultured in Dulbecco's modified Eagle's medium (DMEM) (Hyclone) supplemented with 10\% fetal bovine serum (FBS) (Clark); All cells were cultured at $37^{\circ} \mathrm{C}$ in $5 \% \mathrm{CO}$.

\section{Plasmids and SgRNAs}

All gene silencing was done using a CRISPR-cas9 system, with lentiCRISPR v2 plasmid (Addgene no. 52961). The following sgRNAs were cloned downstream of the U6 promoter: Human, rhesus and mouse METTL3: 5' -GGACACGTGGAGCTCTATCC-3'; Lentiviruses were generated by co-transfection of lentiCRISPR v2 constructs and packaging plasmids (psPAX2, Addgene no. 12260 and pMD2.G, Addgene no. 12259), using PEI DNA transfection reagent (Shanghai maokang biotechnology), into HEK293T cells, according to the manufacturer's instructions. At 48h post transfection, supernatants were collected and filtered through a $0.22 \mu \mathrm{m}$ polyvinylidene fluoride filter (Millex). To induce gene silencing, cells were transduced with lentivirus expressing sgRNA and were puromycin selected immunoblot analysis. 


\section{Virus infections}

Rhesus and simian RV strains, including RRV (Rhesus), SA11-4F (simian), SA11NSP1null (simian) were propagated in MA104 cells as previously described[23]. Viruses were activated by trypsin $(5 \mu \mathrm{g} / \mathrm{ml})$ at $37^{\circ} \mathrm{C}$ for $30 \mathrm{~min}$ prior to infection. Cells were washed with PBS three times and incubated with RV at different MOIs at $37^{\circ} \mathrm{C}$ for $1 \mathrm{hr}$. After removal of RV inoculum, cells were washed with PBS, cultured in serum-free medium (SFM) and harvested for qPCR and western blot analysis.

EW stock virus was prepared by infecting 5-days-old C57BL/6J mice, and harvesting crude centrifugation-clarified intestinal homogenate as previously 372 described[15].

For all rotavirus infection except indicated elsewhere, 8-day-old wild-type mice, or genetically deficient mice were orally inoculated by gavage with RV EW virus as previously described[15]. Mice were sacrificed, stool and small intestinal tissue were collected at indicated time points post infection. Viral loads in intestinal tissues and feces were detected by RT-qPCR.

\section{RT-qPCR}

For cells and tissues, total RNA was extracted with TRNzol Universal reagent (Tiangen) in accordance with the manufacturer's instructions. Real-time PCR was performed using SYBR® Premix Ex Taq $^{\text {TM }}$ II (Tli RNaseH Plus) (Takara) and complementary DNA was synthesized with a PrimeScript ${ }^{\mathrm{TM}}$ RT reagent Kit with gDNA Eraser (Takara). The target genes were normalized to the housekeeping gene (Gapdh, HPRT) shown as 2- $\Delta$ Ct. The used primers are as follows:

Primers detect mouse genes: 
Mettl14-R: AGCCTCTCGATTT CCTCTGT

Fto-F: CTGAGGAAGGAGTGGCATG

Fto-R: TCTCCACCTAAGACTTGTGC

Alkbh5-F: ACAAGATTAGATGCACCGCG

Alkbh5-R: TGTCCATTTCCAGGATCCGG

Wtap-F: GTTATGGCACGGGATGAGTT

397

Wtap-R: ATCTCCTGCTCTTTGGTTGC

Gapdh-F: TGAGGCCGGTGCTGAGTATGTCG

Gapdh-R: CCACAGTCTTCTGGGTGGCAGTG

400

Hprt-F: ACCTCTCGAAGTGTTGGATACAGG

401

Hprt-R: CTTGCGCTCATCTTAGGCTTTG

402

Irf7-F: GCTCCAGTGACTACAAGGCAT

403

Irf7-R: TTGGGAGTTGGGATTCTGAG

404

Isg15-F: GGTGTCCGTGACTAACTCCAT

405

Isg15-R: TGGAAAGGGTAAGACCGTCCT

406

Oas1a-F: GCCTGATCCCAGAATCTATGC

407

Oas1a-R: GAGCAACTCTAGGGCGTACTG

408

Ifnb-F: TCCGAGCAGAGATCTTCAGGAA

409

Ifnb-R: TGCAACCACCACTCATTCTGAG

410

Ifnl3-F: AGCTGCAGGCCTTCAAAAAG

411

Ifnl3-R: TGGGAGTGAATGTGGCTCAG

412

413 Primers detect human genes:

414 hGAPDH-F: ATGACATCAAGAAGGTGGTG

415 hGAPDH-R: CATACCAGGAAATGAGCTTG

416 hIRF7-F: CGAGACGAAACTTCCCGTCC

hIRF7-R: GCTGATCTCTCCAAGGAGCC

hIFNL3-F: TAAGAGGGCCAAAGATGCCTT 
hCXCL10-R: TTGTAGCAATGATCTCAACACG

422

hIFIT1-F: CAACCATGAGTACAAATGGTG

423

hIFIT1-R: CTCACATTTGCTTGGTTGTC

424

425 Primers detect rhesus genes:

426 Rhesus-GAPDH-F: ATGACATCAAGAAGGTGGTG

427 Rhesus-GAPDH-R: CATACCAGGAAATGAGCTTG

428 Rhesus-IFIT1-F: CAACCATGAGTACAAATGGTG

429 Rhesus-IFIT1-R: CTCACACTTGCTTGGTTGTC

430 Rhesus-IRF7-F: GTTCGGAGAGTGGCTCCTTG

431 Rhesus-IRF7-R: TCACCTCCTCTGCTGCTAGG

432 Rhesus-IFNL1-F: ACTCATACGGGACCTGACAT

433 Rhesus-IFNL1-R: GGATTCGGGGTGGGTTGAC

434 Rhesus-IFNb-F: GAGGAAATTAAGCAGCCGCAG

435 Rhesus-IFNb-R: ATTAGCAAGGAAGTTCTCCACA

436

437 Primers detect virus genes:

438 Rotavirus EW-NSP2-F: GAGAATGTTCAAGACGTACTCCA

439 Rotavirus EW-NSP2-R: CTGTCATGGTGGTTTCAATTTC

440 Rotavirus EW-VP4-F: TGGCAAAGTCAATGGCAACG

441 Rotavirus EW-VP4-R: CCGAGACACTGAGGAAGCTG

442 Rotavirus EW-VP7-F: TCAACCGGAGACATTTCTGA

443 Rotavirus EW-VP7-R: TTGCGATAACGTGTCTTTCC

444 RRV VP7-F: ACGGCAACATTTGAAGAAGTC

445 RRV VP7-R: TGCAAGTAGCAGTTGTAACATC

446 RRV NSP2-F: GAGAATCATCAGGACGTGCTT

RRV NSP2-R: CGGTGGCAGTTGTTTCAAT

RRV NSP5-F: CTGCTTCAAACGACCCACTCAC

RRV NSP5-R: TGAATCCATAGACACGCC 


\section{m6A dot blot assay}

Total RNA was isolated from mice ileum using TRNzol Universal Reagent (Tiangen, Lot\#U8825) according to the manufacturer's instructions. RNA samples were quantified using UV spectrophotometry and denatured at $65^{\circ} \mathrm{C}$ for $5 \mathrm{~min}$. The m6A-dot-blot was performed according to a published work[37]. In brief, the primary rabbit anti-m6A antibody (1:5000, Synaptic System, \#202003) was applied to the Amersham Hybond-N+ membrane (GE Healthcare, USA) containing RNA samples. Dot blots were visualized by the imaging system after incubation with secondary antibody HRP-conjugated Goat anti-rabbit IgG (Beyotime, A0208).

\section{Western blot}

Briefly, cells and tissue were lysed with RIPA buffer (Beyotime Biotechnology) supplemented with PMSF (Beyotime Biotechnology) and protease inhibitor cocktail (Roche). Mettl3 (abcam, ab195352, 1:2000), Mettl14 (sigma, HPA038002, 1:2000), ALKBH5 (sigma, HPA007196, 1:2000), FTO (abcam, ab92821), NSP1 and VP6 (gift from Harry B. Greenberg lab), Gapdh (proteintech), and betaactin(proteintech) antibodies were used in accordance with the manufacturer's instructions. After incubation with the primary antibody overnight, the blotted PVDF membranes (Immobilon, IPVH00010) were incubated with goat anti-rabbit IgG-HRP (Beyotime, A0208) or goat anti-mouse IgG-HRP (Beyotime, A0216) and exposed with BIO-RAD ChemiDocTM Imaging System for a proper exposure period.

\section{RNA degradation assay}

The stability of targeted mRNA was assessed as previously described[5]. In brief, Mettl3 knock down HT-29 and control cell were plated on 24-well plate. Actinomycin-D (MCE, HY17559) was added to a final concentration of $5 \mu \mathrm{M}$, and cells were harvested by indicated time points after actinomycin-D treatment. The RNA samples are processed and qPCR was used to measure the mRNA transcripts, all data were normalized to that of $\mathrm{t}=0$ time point. 


\section{Dual-luciferase assay}

483 pmirGLO (Firefly luciferase, hRluc) vector of the Dual-luciferase Reporter assay system (Promega, E1910) was used to determine the function of m6A modification within the 3'UTR of Irf7 transcripts. The potential m6A modification sites were predicted on SRAMP website. The assay was performed according to the manufacture's instruction: Briefly, 300 ng of pmirGLO vector containing Irf73'UTR or m6A-mutant Irf7-3'UTR were transfected into HEK293T cells in triplicate wells. The relative luciferase activity was accessed $36 \mathrm{~h}$ post-transfection.

\section{Isolation of IECs in the intestine}

492

493

494

495

496

497

498

499

500

501

502

503

504

505

506

507

508

509

Small intestines were excised and flushed thoroughly three times with PBS. They were turned inside out and cut into $\sim 1 \mathrm{~cm}$ sections then transferred into RPMI with $2 \mathrm{mM}$ EDTA, and shaken for $20 \mathrm{~min}$ at $37^{\circ} \mathrm{C}$. Supernatants were collected through a 100-mm cell strainer to get single-cell suspensions. Cells were collected as the IEC fraction which contains both epithelial cells ( 90\%) and lymphocytes (IEL, 10\%). Single-cell suspension was used for further analysis.

\section{RNA-Seq}

IECs from Mettl3 ${ }^{\Delta \mathrm{IEC}}$ mice as well as the wild-type littermate control mice were isolated as described in previous section. Total RNAs were extracted with TRNzol universal RNA Reagen kits. Berrygenomics (Beijing, China) processed the total RNA and constructed the mRNA libraries, and subject them to standard illumine sequencing on Novaseq 6000 system, and obtained > 40 million Pair-end 150 reads for each sample. Raw RNA-sequencing reads were aligned to the mouse genome (mm10, GRCm38) with STAR (v2.5.3a). Gene expression levels and differential analysis was performed with edgeR(v3.29.2). Genes were considered significantly differentially expressed if showing $\geq 1.5$-fold change and FDR $<0.05$.

\footnotetext{
Gene set analysis was performed and enriched pathways were obtained through
} 
510 online bioinformatics tools (metascape) and GSEA (v4.0.3). Pathway plot were

511 gene-rated with R package 'ggplot2'[5].

\section{m6A RNA-IP-qPCR \& m6A RNA-IP-Seq}

m6A RNA-IP-Seq was carried out according to a previously published protocol[5]. In brief, total cellular RNA extracted from WT C57 mice IEC was fragmented by $\mathrm{ZnCl} 2$ followed by ethanol precipitation. Fragmented RNA was incubated with an anti-m6A antibody (Sigma Aldrich ABE572) and IgG IP Grade Rabbit polyclonal antibody (abcam, lot: 934197). The eluted RNA and input were subjected to highthroughput sequencing using standard protocols (Illumina, San Diego, CA, USA) or processed as described in 'RT-qPCR' section, except that the data were normalized to the input samples. The m6A RIP-Seq data were analyzed as described previously[5].

RIP-ptpn4-F: CCTCCCATCCCGGTCTCCACC

RIP-ptpn4-R: GGCTGCCCATCTTCAGGGGT

RIP-RPS14-F: ACCTGGAGCCCAGTCAGCCC

RIP-RPS14-R: CACAGACGGCGACCACGACG

m6A-IRF7-F: GACAGCAGCAGTCTCGGCTT m6A-IRF7-R: ACCCAGGTCCATGAGGAAGT m6A sites on RV-EW RNA were predicted on http: //www.cuilab.cn/sramp website, and m6A-RIP-qPCR primer were designed on NCBI primer blast according to the predicted $\mathrm{m} 6 \mathrm{~A}$ sites. m6A-RIP-qPCR primer:

RIP-EW-VP1-R: AACCTGTCCGTCAACCATTC 
RIP-EW-VP4-R: GGCTGAGATGTCATCGAAGTT

RIP-EW-NSP1-F: CCTCACATCTCTGCTACATGAACT

RIP-EW-NSP1-R: TGCTGGTTGGACATGGAATGA

RIP-EW-VP6-F: CTGCACTTTTCCCAAATGCTCA

RIP-EW-VP6-R: GAGTCAATTCTAAGTGTCAGTCCG

RIP-EW-NSP3-F: CTTGACGTGGAGCAGCAAC

RIP-EW-NSP3-R: AATGTTTCAATGTCGTCCAACG

RIP-EW-NSP2-F: TCCACCACTCTAAAGAACTACTGC

RIP-EW-NSP2-R: TCCGCTGTCATGGTGGTTTC

RIP-EW-VP7-F: TCGGAACTTGCAGACTTGAT

RIP-EW-VP7-R: GCTTCGTCTGTTTGCTGGTA

RIP-EW-NSP4-F: TGCACTGACTGTTCTATTTACGA

RIP-EW-NSP4-R: GGGAAGTTCGCATTGCTAGT

RIP-EW-NSP5/6-F: GGACACCGCAAGGTCAAAAA

RIP-EW-NSP5/6-R: TCGTCTGAGTCTGATTCTGCTT

\section{J2 Immunofluorescent staining}

IECs from Mettl3 $3^{\triangle I E C}$ mice as well as from the wild-type littermate control mice were isolated as described in previous section. Isolated IEC were centrifuged onto glass slides and fixed with 4\% Paraformaldehyde for $30 \mathrm{~min}$ at room temperature. Subsequently, permeabilized and blocked with PBS containing 0.1\% Triton-X-100 and $5 \%$ bovine serum albumin for $1 \mathrm{~h}$ at room temperature. Double-stranded RNA (dsRNA) was labeled by a mouse monoclonal antibody J2 (Scisons) for $2 \mathrm{~h}$ at room temperature, followed by incubation with anti-mouse IgG Alexa Fluor 594conjugated antibody (Invitrogen) for $1 \mathrm{~h}$, and cells nuclei were visualized with 4,6diamidino-2-phenylindole (DAPI, Invitrogen). All fluorescence images were analyzed via confocal imaging using Zeiss LSM880.

\section{Statistical analysis}

Statistical analysis was performed with the GraphPad Prism 8.0 (GraphPad, Inc., 
USA). Experiments were independently repeated for indicated times listed in the figure legend. Representative data was exhibited as the means \pm SEM. Quantitative data was compared using two-tail Student t test. In addition, correlational analysis of gene expression was conducted with linear regression. P-values for every result were labeled on figures, and $\mathrm{P}<0.05$ was reckoned as statistically significant $\left({ }^{*} \mathrm{P}<0.05,{ }^{* *} \mathrm{P}<0.01,{ }^{* * *} \mathrm{P}<0.001,{ }^{* * * *} \mathrm{P}<0.0001\right.$, NS., not significant $)$.

\section{Data availability statement}

The data that support the findings of this study are available from the corresponding author upon reasonable request. RNA sequencing data are available from the SRA database with accession numbers PRJNA713535.

\section{Author Contribution:}

A.W. designed, performed and interpreted experiments. W.T. analyzed the RNA sequencing data and m6A-Seq data. J.T. did the m6A-Seq experiment. J.W., J.G., G.Z., X.R., R.L., and D.W. helped with animal experiments and cellular experiments., S.D., R.A.F., K.Z., and W.P. provided critical comments and suggestions; A.W. and S.Z. wrote the manuscript. S.D. and W.T. edited the manuscript. S.Z. and H.B.L. supervised the project.

\section{Acknowledgements}

We would like to thank Hongdi Ma, Taidou Hu, Kaixin He, Yinglei Wang, Ji Hu, Anlei Wang, and Meng Guo for technical help and helpful discussion.

\section{Funding}

This work was supported by grants from the Strategic Priority Research Program of the Chinese Academy of Sciences (XDB29030101)(SZ), the National Key R\&D 
600

601

602

603

Program of China (2018YFA0508000)(SZ), and National Natural Science Foundation of China (81822021, 91842105, 31770990, 82061148013, 81821001)(SZ).

\section{Competing interests: The authors declare no competing interests.}

\section{References:}

[1]. Roundtree, I.A., et al., Dynamic RNA Modifications in Gene Expression Regulation. Cell, 2017. 169(7): p. 1187-1200.

[2]. Xu, W., et al., METTL3 regulates heterochromatin in mouse embryonic stem cells. Nature, 2021.

[3]. Chelmicki, T., et al., m(6)A RNA methylation regulates the fate of endogenous retroviruses. Nature, 2021.

[4]. Tsai, K., D.G. Courtney and B.R. Cullen, Addition of m6A to SV40 late mRNAs enhances viral structural gene expression and replication. PLoS Pathog, 2018. 14(2): p. e1006919.

[5]. Li, H.B., et al., m(6)A mRNA methylation controls $\mathrm{T}$ cell homeostasis by targeting the IL7/STAT5/SOCS pathways. Nature, 2017. 548(7667): p. 338-342.

[6]. Hesser, C.R., et al., N6-methyladenosine modification and the YTHDF2 reader protein play cell type specific roles in lytic viral gene expression during Kaposi's sarcoma-associated herpesvirus infection. PLoS Pathog, 2018. 14(4): p. e1006995.

[7]. Imam, H., et al., N6-methyladenosine modification of hepatitis B virus RNA differentially regulates the viral life cycle. Proc Natl Acad Sci U S A, 2018. 115(35): p. 8829-8834.

[8]. Ye, F., E.R. Chen and T.W. Nilsen, Kaposi's Sarcoma-Associated Herpesvirus Utilizes and Manipulates RNA N(6)-Adenosine Methylation To Promote Lytic Replication. J Virol, 2017. 91(16).

[9]. Liu, Y., et al., N (6)-methyladenosine RNA modification-mediated cellular metabolism rewiring inhibits viral replication. Science, 2019. 365(6458): p. 1171-1176.

[10]. Gao, Y., et al., m(6)A Modification Prevents Formation of Endogenous Double-Stranded RNAs and Deleterious Innate Immune Responses during Hematopoietic Development. Immunity, 2020. 52(6): p. 1007-1021.e8.

[11].Hao, H., et al., N6-methyladenosine modification and METTL3 modulate enterovirus 71 replication. Nucleic Acids Res, 2019. 47(1): p. 362-374. 
[12]. Rubio, R.M., et al., RNA m(6) A modification enzymes shape innate responses to DNA by regulating interferon beta. Genes Dev, 2018. 32(23-24): p. 1472-1484.

[13]. Winkler, R., et al., m(6)A modification controls the innate immune response to infection by targeting type I interferons. Nat Immunol, 2019. 20(2): p. 173-182.

[14]. Crawford, S.E., et al., Rotavirus infection. Nat Rev Dis Primers, 2017. 3: p. 17083.

[15]. Ding, S., et al., Rotavirus VP3 targets MAVS for degradation to inhibit type III interferon expression in intestinal epithelial cells. Elife, 2018. 7.

[16]. Barro, M. and J.T. Patton, Rotavirus NSP1 inhibits expression of type I interferon by antagonizing the function of interferon regulatory factors IRF3, IRF5, and IRF7. J Virol, 2007. 81(9): p. 4473-81.

[17]. Honda, K., et al., IRF-7 is the master regulator of type-I interferon-dependent immune responses. Nature, 2005. 434(7034): p. 772-7.

[18]. Lin, J.D., et al., Distinct Roles of Type I and Type III Interferons in Intestinal Immunity to Homologous and Heterologous Rotavirus Infections. PLoS Pathog, 2016. 12(4): p. e1005600.

[19]. Pott, J., et al., IFN-lambda determines the intestinal epithelial antiviral host defense. Proc Natl Acad Sci U S A, 2011. 108(19): p. 7944-9.

[20]. Du J, et al., Detailed analysis of BALB/c mice challenged with wild type rotavirus EDIM provide an alternative for infection model of rotavirus. Virus Res, 2017. 228: p. 134-140.

[21]. Ciancanelli, M.J., et al., Host genetics of severe influenza: from mouse Mx1 to human IRF7. Curr Opin Immunol, 2016. 38: p. 109-20.

[22]. Barro, M. and J.T. Patton, Rotavirus nonstructural protein 1 subverts innate immune response by inducing degradation of IFN regulatory factor 3. Proc Natl Acad Sci U S A, 2005. 102(11): p. 4114-9.

[23]. Ding, S., et al., Comparative Proteomics Reveals Strain-Specific beta-TrCP Degradation via Rotavirus NSP1 Hijacking a Host Cullin-3-Rbx1 Complex. PLoS Pathog, 2016. 12(10): p. e1005929.

[24]. Kanai, Y., et al., Entirely plasmid-based reverse genetics system for rotaviruses. Proc Natl Acad Sci U S A, 2017. 114(9): p. 2349-2354.

[25]. Brocard, M., A. Ruggieri and N. Locker, m6A RNA methylation, a new hallmark in virus-host interactions. J Gen Virol, 2017. 98(9): p. 2207-2214.

[26]. Lavi, S. and A.J. Shatkin, Methylated simian virus 40-specific RNA from nuclei and cytoplasm of infected BSC-1 cells. Proc Natl Acad Sci U S A, 1975. 72(6): p. 2012-6.

[27]. Krug, R.M., M.A. Morgan and A.J. Shatkin, Influenza viral mRNA contains internal N6methyladenosine and 5'-terminal 7-methylguanosine in cap structures. J Virol, 1976. 20(1): p. 45-53.

[28]. Sommer, S., et al., The methylation of adenovirus-specific nuclear and cytoplasmic RNA. Nucleic Acids Res, 1976. 3(3): p. 749-65. 
661

662

663

664

665

666

667

668

669

670

671

672

673

674

675

676

677

678

679

680

681

682

683

684

685

686

687

688

689

690

[29]. Dimock, K. and C.M. Stoltzfus, Sequence specificity of internal methylation in B77 avian sarcoma virus RNA subunits. Biochemistry, 1977. 16(3): p. 471-8.

[30]. Kane, S.E. and K. Beemon, Precise localization of m6A in Rous sarcoma virus RNA reveals clustering of methylation sites: implications for RNA processing. Mol Cell Biol, 1985. 5(9): p. 2298-306.

[31]. Gokhale, N.S., et al., N6-Methyladenosine in Flaviviridae Viral RNA Genomes Regulates Infection. Cell Host Microbe, 2016. 20(5): p. 654-665.

[32]. Lichinchi, G., et al., Dynamics of Human and Viral RNA Methylation during Zika Virus Infection. Cell Host Microbe, 2016. 20(5): p. 666-673.

[33]. Sanchez-Tacuba, L., et al., An Optimized Reverse Genetics System Suitable for Efficient Recovery of Simian, Human, and Murine-Like Rotaviruses. J Virol, 2020. 94(18).

[34]. Chen, S., et al., N6-methyladenosine modification of HIV-1 RNA suppresses type-I interferon induction in differentiated monocytic cells and primary macrophages. PLoS Pathog, 2021. 17(3): p. e1009421.

[35]. Kim, G.W., et al., N (6)-Methyladenosine modification of hepatitis B and C viral RNAs attenuates host innate immunity via RIG-I signaling. J Biol Chem, 2020. 295(37): p. 13123-13133.

[36]. Zhou, J., et al., m(6)A demethylase ALKBH5 controls CD4(+) T cell pathogenicity and promotes autoimmunity. Sci Adv, 2021. 7(25).

[37]. Shen, L., et al., N(6)-Methyladenosine RNA Modification Regulates Shoot Stem Cell Fate in Arabidopsis. Dev Cell, 2016. 38(2): p. 186-200.

\section{Figure Legends}

Figure 1. Rotavirus infection increases global m6A modifications, and Mettl3 deficiency in intestinal epithelial cells results in increased resistance to rotavirus infection.

(a) m6A dot blot analysis of total RNA in ileum tissues from mice with different ages. Methylene blue (MB) staining was the loading control.

(b) Quantitative analysis of (a) (mean \pm SEM), statistical significance was determined by Student's t-test ( ${ }^{* *} \mathrm{P}<0.005,{ }^{* * *} \mathrm{P}<0.001$, NS., not significant). The quantitative m6A signals were normalized to quantitative MB staining signals. 
691 (c) qPCR analysis of indicated genes in ileum tissues from mice with different ages 692 (mean \pm SEM). Statistical significance was determined by Student's t-tests 693 between groups ( ${ }^{*} \mathrm{P}<0.05,{ }^{* * *} \mathrm{P}<0.001$, NS., not significant).

694 (d) WT mice were infected by rotavirus EW strain at 8 days post birth. m6A dot 695 blot analysis of total RNA in ileum tissue at 2 dpi. BMDMs from Wild type (WT) 696 and Mettl3 KO were used as the positive and negative controls, respectively.

697

698

699

700

701

702

703

704

705

706

707

708

709

710

711

712 Methylene blue (MB) staining was the loading control.

(e) Quantitative analysis of (c) (mean \pm SEM). Statistical significance was determined by Student's t-test $\left({ }^{* *} \mathrm{P}<0.005\right)$. The quantitative $\mathrm{m} 6 \mathrm{~A}$ signals were normalized to quantitative MB staining signals.

(f-g) Mettl $3^{\triangle \mathrm{IEC}}$ mice and littermate controls were infected by rotavirus EW strain at 8 days post birth. qPCR analysis of RV viral loads in ileum tissue (f) or fecal samples (g) from $\mathrm{Mettl}^{\triangle}{ }^{\mathrm{IEC}}$ mice and littermate controls at 4 days post infection (dpi) (littermate WT $\mathrm{n}=4$, Mettl $^{\triangle}{ }^{\mathrm{IEC}} \mathrm{n}=4$, mean \pm SEM). Statistical significance was determined by Student's t-tests between genotypes ( $\left.* \mathrm{P}<0.05,{ }^{* * *} \mathrm{P}<0.001\right)$.

(h) qPCR analysis of indicated genes in Rhesus rotavirus (RRV)-infected HT-29 cells transduced with Mettl3 sgRNA or control sgRNA, at indicated hours post infection (hpi) (mean \pm SEM), statistical significance was determined by Student's t-test $\left({ }^{*} \mathrm{P}<0.05,{ }^{* * *} \mathrm{P}<0.001, \mathrm{NS}\right.$., not significant).

Experiments in (a-e, and $\mathbf{h}$ ) are repeated twice, (f and $\mathbf{g}$ ) are repeated four times.

Figure 2. Mettl3 deficiency in intestinal epithelial cells results in decreased m6A deposition on Irf7, and increased interferon responses.

(a) Gene ontology (GO) analysis of differentially expressed genes in IECs from Mettl ${ }^{\triangle}{ }^{\triangle}$ IEC mice vs IECs from littermate WT mice.

(b) Heat map of a subset of up-regulated ISGs in IECs from Mettl ${ }^{\triangle}{ }^{\mathrm{IEC}}$ mice vs IECs 
717 from littermate WT mice, as revealed by RNA-seq (normalized data).

718 (c) m6A-RIP-seq analysis of Irf7 and Gapdh mRNA in the ileum of WT mice.

719 (d) Gene regulation network of a subset of up-regulated genes including IRF7.

(e) Heat map of Interferon regulatory factors (Irfs) in IECs from Mettl $3^{\triangle}$ IEC mice vs

IECs from littermate WT mice, as revealed by RNA-seq (RPKM).

(f) $M e t t l{ }^{\triangle}{ }^{\triangle}$ IEC mice and littermate controls were infected by EW at 8 days post birth. qPCR analysis of the Irf7 expression in ileum and jejunum from Mettl3 ${ }^{\triangle}{ }^{\mathrm{IEC}}$ mice and littermate control at 2 dpi (littermate WT $n=4, M \operatorname{ctt} 3^{\triangle}{ }^{\mathrm{IEC}}$ mice $\mathrm{n}=3$, mean \pm SEM). Statistical significance was determined by Student's t-test $\left({ }^{*} \mathrm{P}<0.05,{ }^{* *} \mathrm{P}<\right.$ $0.005)$.

(g) q-PCR analysis of Irf7 mRNA in Mettl3 knockdown HT-29 cells or control cells in indicated time points post actinomycin $D$ treatment $(n=3$, mean \pm SEM). Statistical significance was determined by Student's t-test $\left({ }^{*} \mathrm{P}<0.05,{ }^{* * *} \mathrm{P}<0.001\right.$, NS., not significant).

(h) Relative luciferase activity of HEK293T cells transfected with pmirGLO-Irf73'UTR (Irf7-WT) or pmirGL0-Irf7-3'UTR containing mutated m6A modification sites (Irf7-MUT). The firefly luciferase activity was normalized to Renilla luciferase activity. Statistical significance was determined by Student's t-test $\left({ }^{*} \mathrm{P}<0.05\right)$.

(i) $M e t t l 3^{\triangle}$ IEC mice and littermate control and were infected by EW at 8 days post birth. qPCR analysis of selected IFNs and ISGs in ileum tissue at 2 dpi (littermate WT, $n=4, M^{2} t t l 3^{\triangle}$ IEC mice, $n=3$, mean \pm SEM). Statistical significance was determined by Student's t-tests between genotypes $\left({ }^{*} \mathrm{P}<0.05\right.$, ${ }^{* *} \mathrm{P}<0.005$, ${ }^{* * *} \mathrm{P}<$ 0.001). 
(a-c) WT control mice, Mettl3 ${ }^{\triangle \mathrm{IEC}}$ mice, Irf7 /- mice and Mettl3 ${ }^{\Delta \mathrm{IEC}}$ Irf7 ${ }^{-/-}$mice are all littermates. They were infected by RV EW at 8 days post birth. qPCR analysis of selected IFNs (a), ISGs (b), or Irf7 (c) expression in ileum from indicated groups of

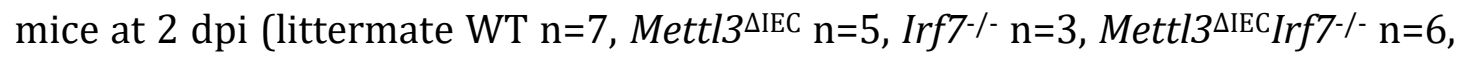
mean \pm SEM). Statistical significance was determined by Student's t-tests between genotypes $\left({ }^{*} \mathrm{P}<0.05,{ }^{* *} \mathrm{P}<0.005,{ }^{* * *} \mathrm{P}<0.001,{ }^{* * *} \mathrm{P}<0.0001, \mathrm{NS}\right.$., not significant). (littermate WT n=5, Mettl3 ${ }^{\Delta \mathrm{IEC}} \mathrm{n}=5, \operatorname{Irf7-/-} \mathrm{n}=3$, Mettl3 $^{\Delta \mathrm{IEC}} \operatorname{Irf7-/-n=4,~mean~} \pm \mathrm{SEM}$ ). $<0.05$, NS., not significant).

(e-f) qPCR analysis of RV proteins expression (e) or Mettl3 and Irf7 (f) in ileum from indicated groups of mice at 4 dpi (littermate WT n=7, Mettl3 ${ }^{\Delta \mathrm{IEC}} \mathrm{n}=5$, Irf7-/$\mathrm{n}=3$, Mettl3 $^{\mathrm{AIEC}} \operatorname{Irf7} 7^{-/-} \mathrm{n}=6$, mean $\left.\pm \mathrm{SEM}\right)$. Statistical significance was determined by Student's t-tests between genotypes and $\left({ }^{*} \mathrm{P}<0.05,{ }^{* *} \mathrm{P}<0.005,{ }^{* * *} \mathrm{P}<0.001\right.$, NS., not significant).

Experiments in (a-f) are repeated twice.

Figure 4. Rotavirus suppresses ALKBH5 expression through NSP1 to evade

(a) WT mice were infected by RV EW at 8 days post birth. Immunoblotting with antibodies target ALKBH5, FTO, METTL14 and METTL3 in ileum tissue from mice infected with RV EW at 2 dpi or treated with PBS.

(b) Quantitative analysis of (a)(mean \pm SEM), Statistical significance was determined by Student's t-test $(* \mathrm{P}<0.05$, NS., not significant). 
769 post birth. qPCR analysis of indicated genes expression in ileum (c), viral shedding

770 in feces (d), and viral proteins expression in ileum (e), from $A l k b h 5^{\triangle}$ IEC mice or

771 littermate controls at 4 days post infection (littermate WT $\mathrm{n}=6, A l k b h 5^{\triangle}{ }^{\mathrm{IEC}} \mathrm{n}=5$,

772 mean \pm SEM). Statistical significance was determined by Student's t-tests between

773 genotypes $\left({ }^{*} \mathrm{P}<0.05, \mathrm{NS} .\right.$, not significant $)$.

774 (f) Immunoblotting with antibodies target ALKBH5, NSP1, VP6 and GAPDH in 775 HEK293 cells infected by SA11-4F and SA11-NSP1 null (MOI=1) for 24h.

776 (g) Graphical abstract illustrating the functions and molecular mechanisms of 777 m6A modifications on Irf7 in anti-RV infection.

778 Experiments in (a-e) are repeated three time, (f) are repeated twice. 
a

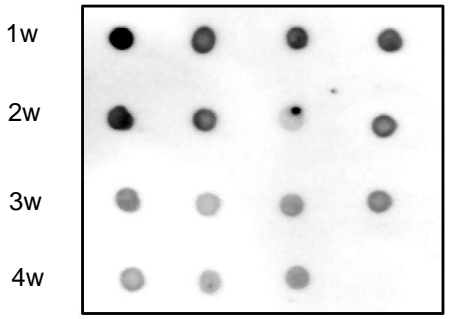

anti-m6A methylene blue
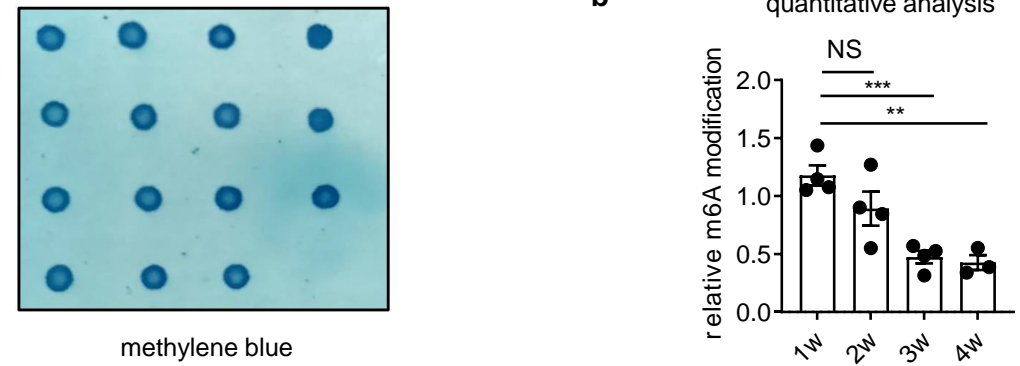

C

Alkbh5 in ileum

Fto in ileum

Mett/3 in ileum
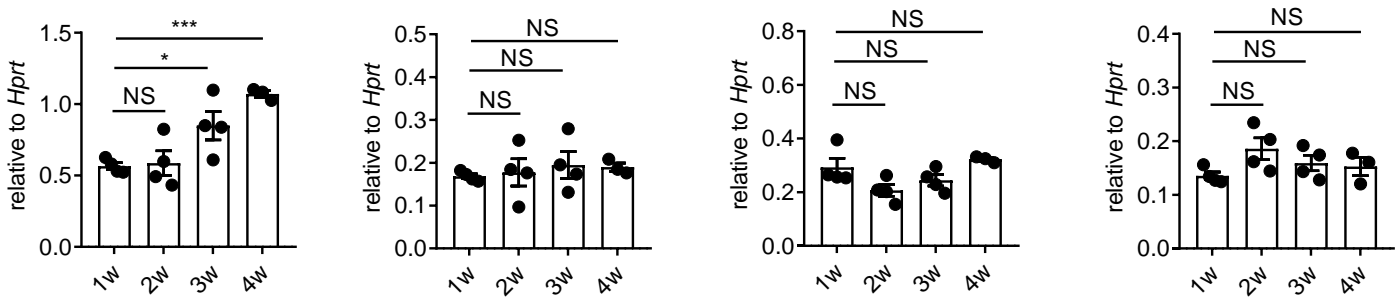

quantitative analysis

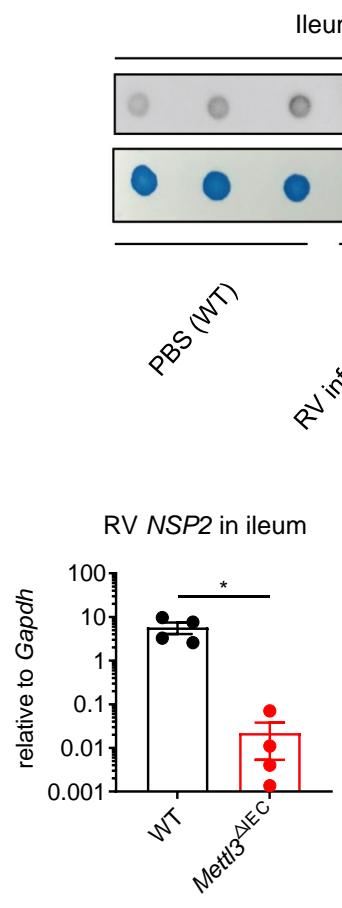

leum

BMDM

anti-m6A

methylene blue

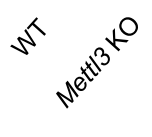

f
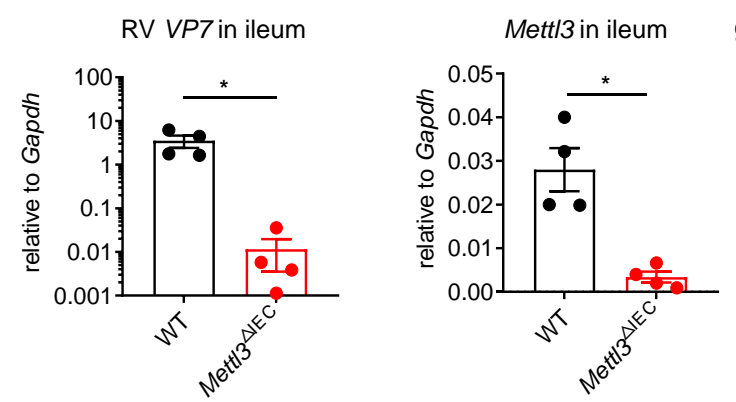

h

NSP2 in HT29

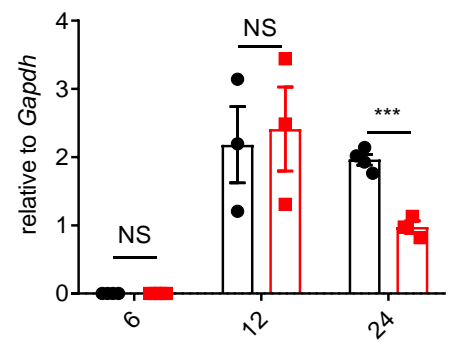

- sgEV

- sgMett/3

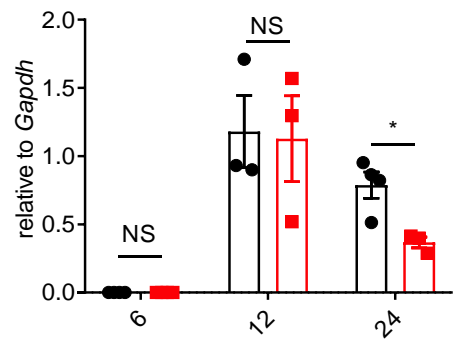

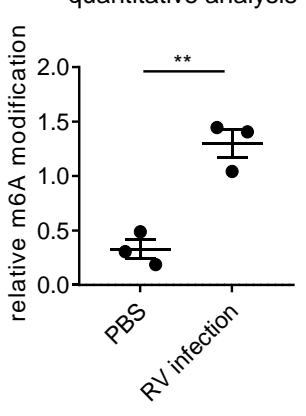

RV VP4 in feces

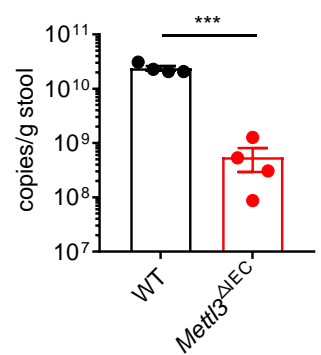

- sgEV

- sgMett/3

Figure 1. Rotavirus infection increases global m6A modifications, and Mett/3 deficiency in intestinal epithelial cells results in increased resistance to rotavirus infection. 
a

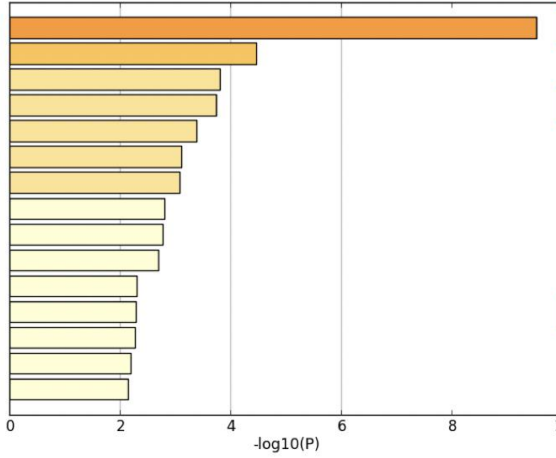

Fold $>2$, FDR $<0.1$
GO:0051607: defense response to virus

GO:1901661: quinone metabolic process

R-MMU-8978868: Fatty acid metabolism

GO:0035456: response to interferon-beta

GO:1990542: mitochondrial transmembrane transport

GO:0001666: response to hypoxia

GO:1901615: organic hydroxy compound metabolic process

GO:0098754: detoxification

GO:0070169: positive regulation of biomineral tissue development

GO:0050873: brown fat cell differentiation

GO:0044257: cellular protein catabolic process

GO:0045089: positive regulation of innate immune response

R-MMU-174824: Plasma lipoprotein assembly, remodeling, and clearance

GO:0072521: purine-containing compound metabolic process

GO:0031333: negative regulation of protein complex assembly b

ISGs in RNA-seq
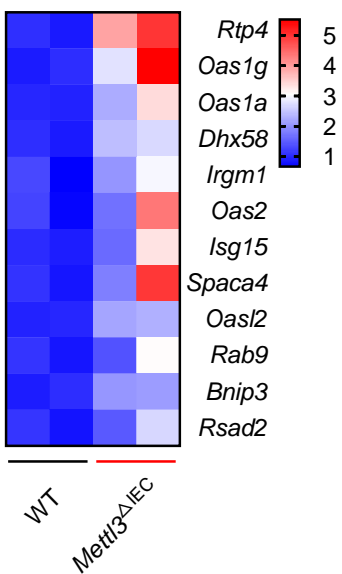

C

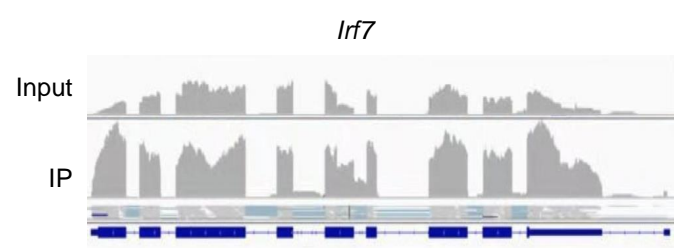

Gapdh

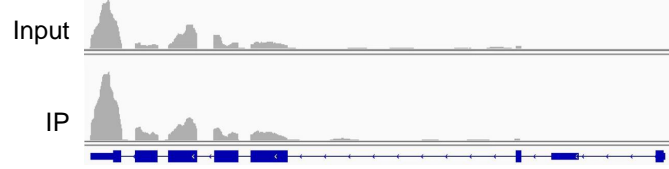

f

Irf7 expression in mice ileum

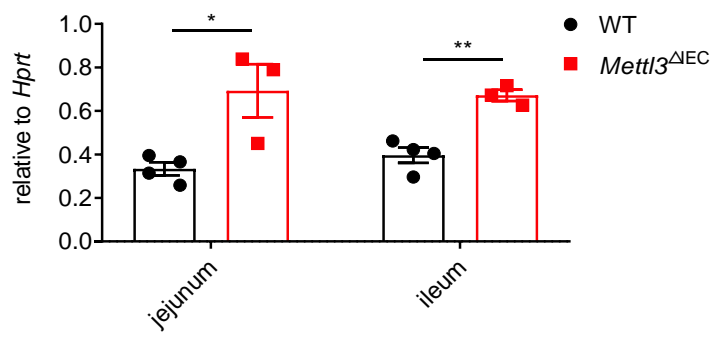

i
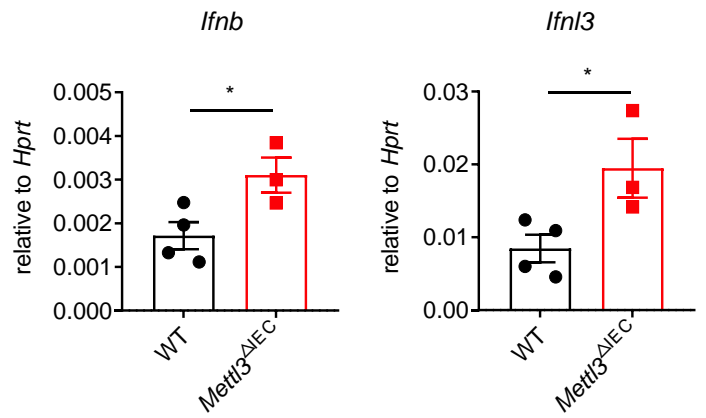

d

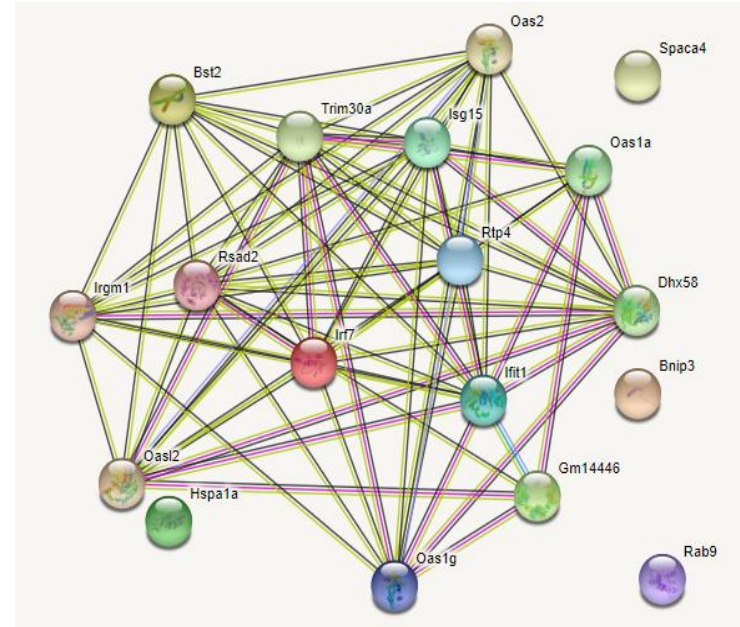

g

Irf7 expression in HT29

h
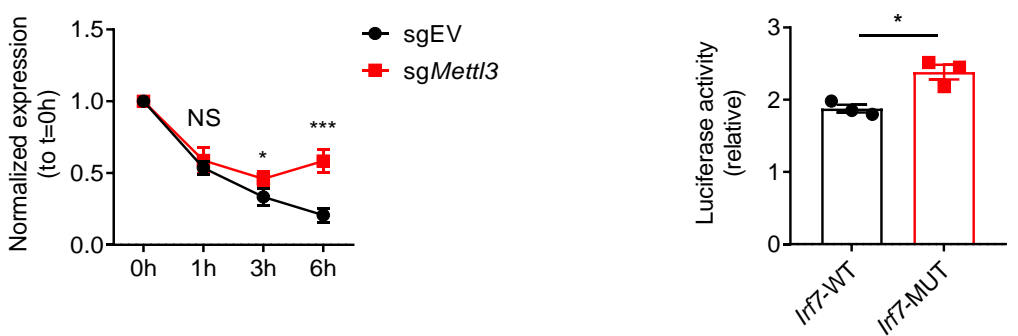

Oas1a

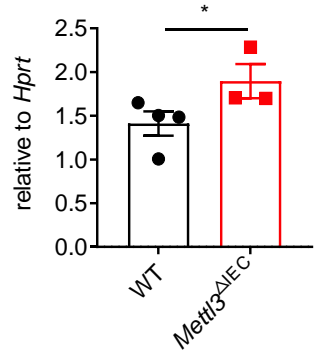

Irfs in RNA-seq e

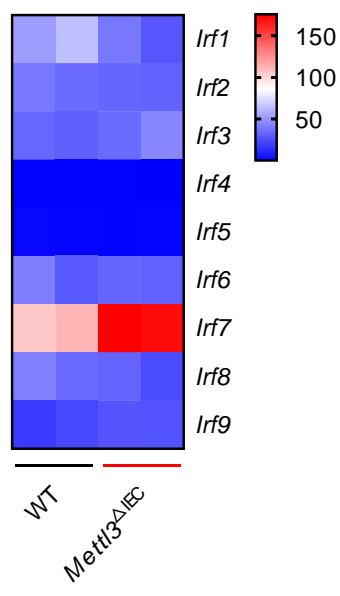

Figure 2. Mett/3 deficiency in intestinal epithelial cells results in decreased m6A deposition on Irf7, and increased interferon responses. 
a

Ifnb in ileum

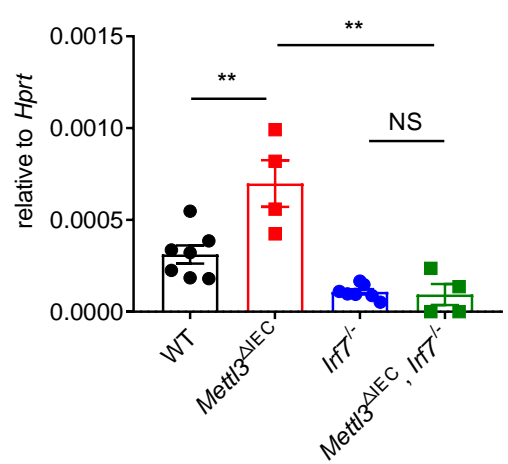

c

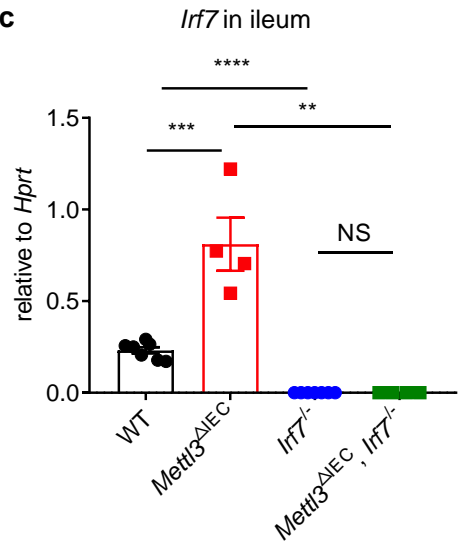

e

NSP2 in ileum

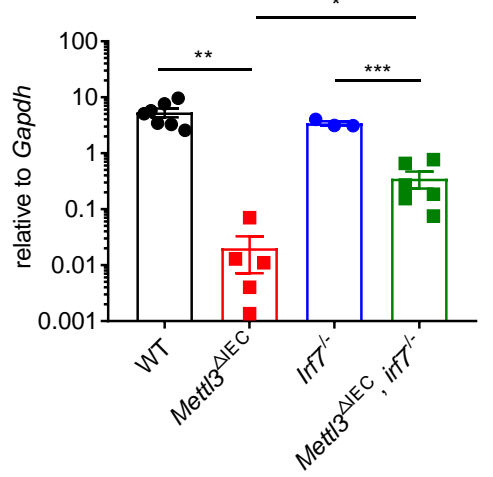

Ifn/3 in ileum

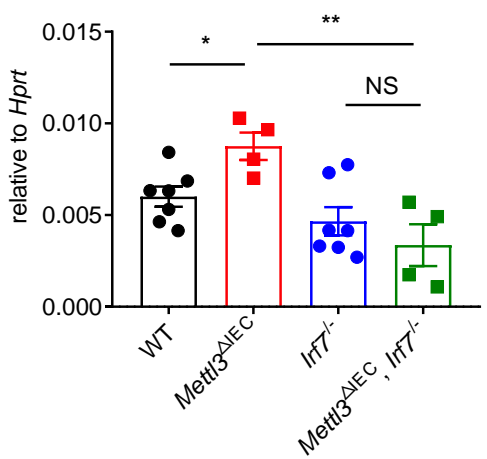

d $\quad$ VP4 in mice feces

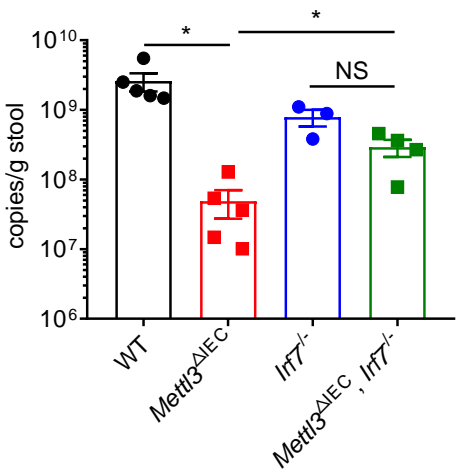

VP7 in ileum

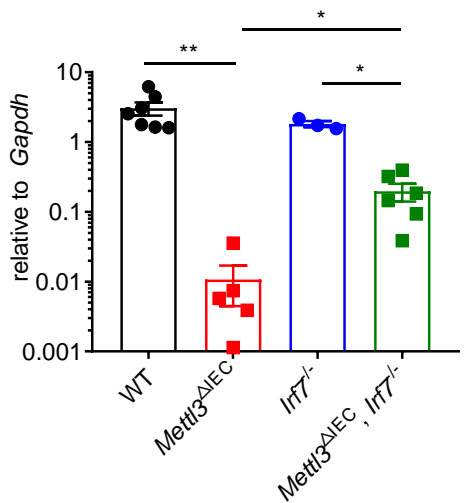

b

Isg15 in ileum

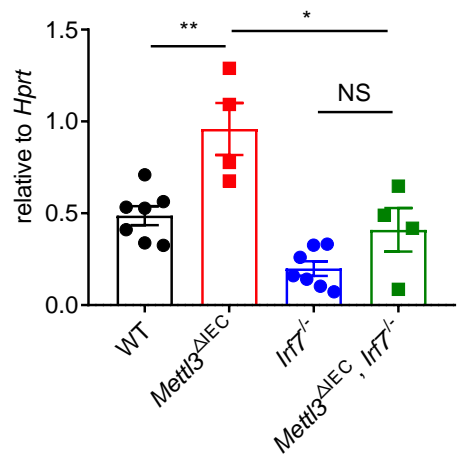

VP7 in mice feces

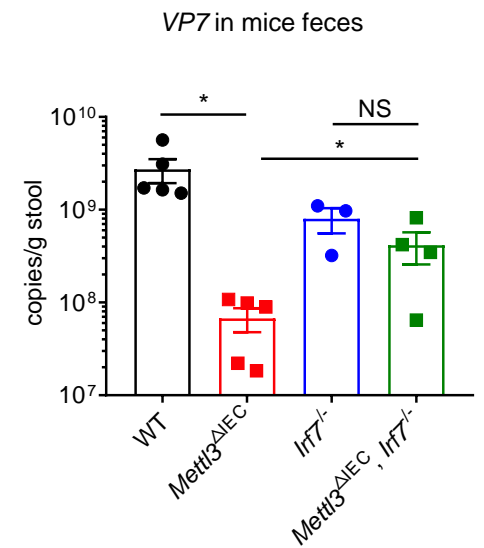

f

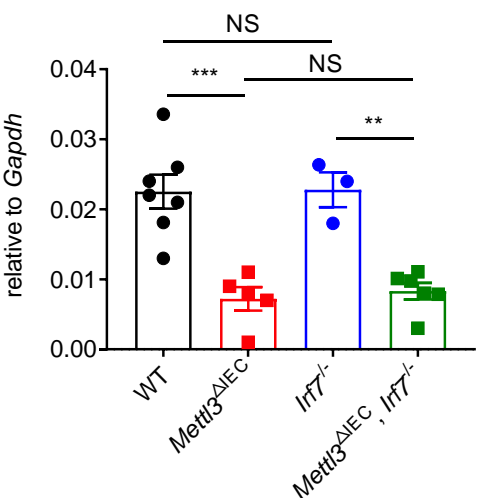

Mett/3 in IEC
Ifit 1 in ileum

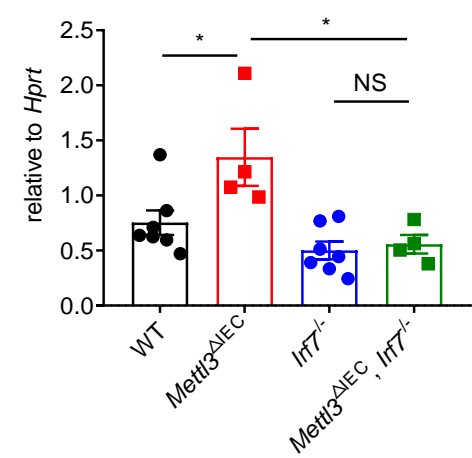

Irf7 in ileum

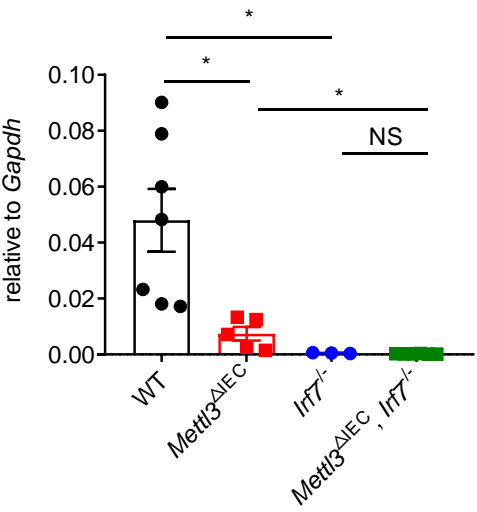

Figure 3. IRF7 Deficiency attenuated the increased interferon response and resistance to rotavirus infection in Mett/3دIEC mice. 


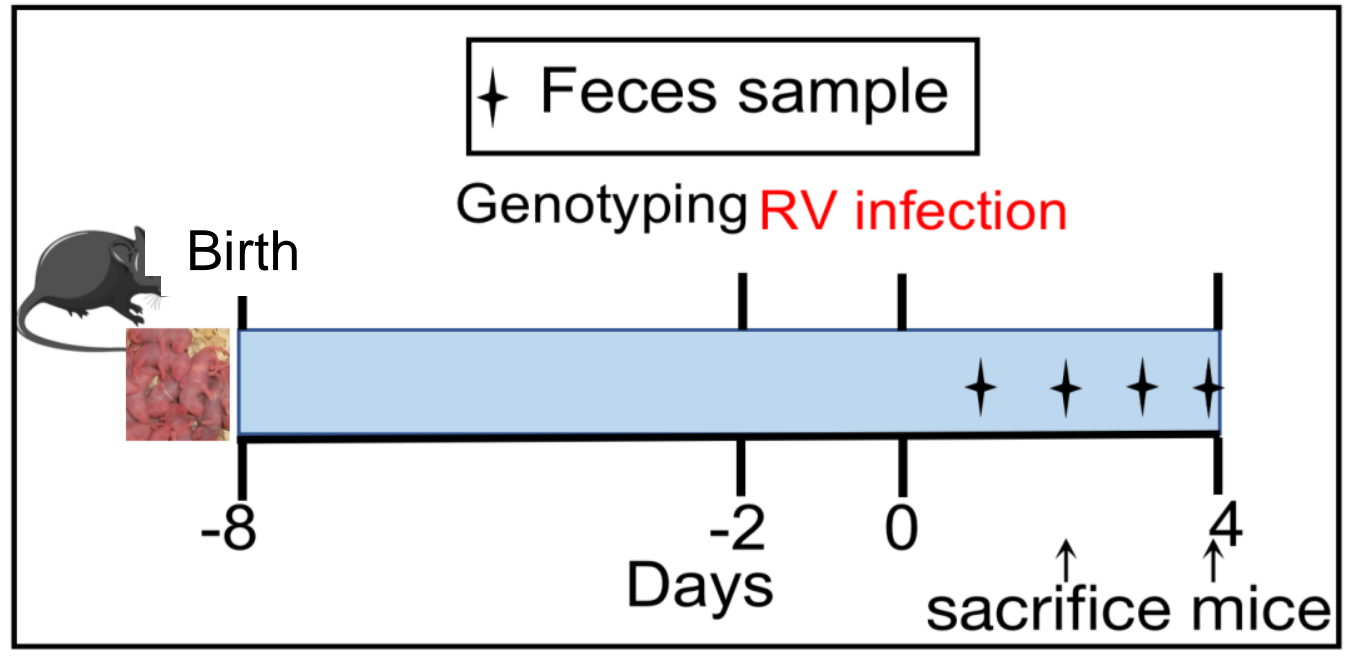

Figure s1. Schematic design of RV infection 
a

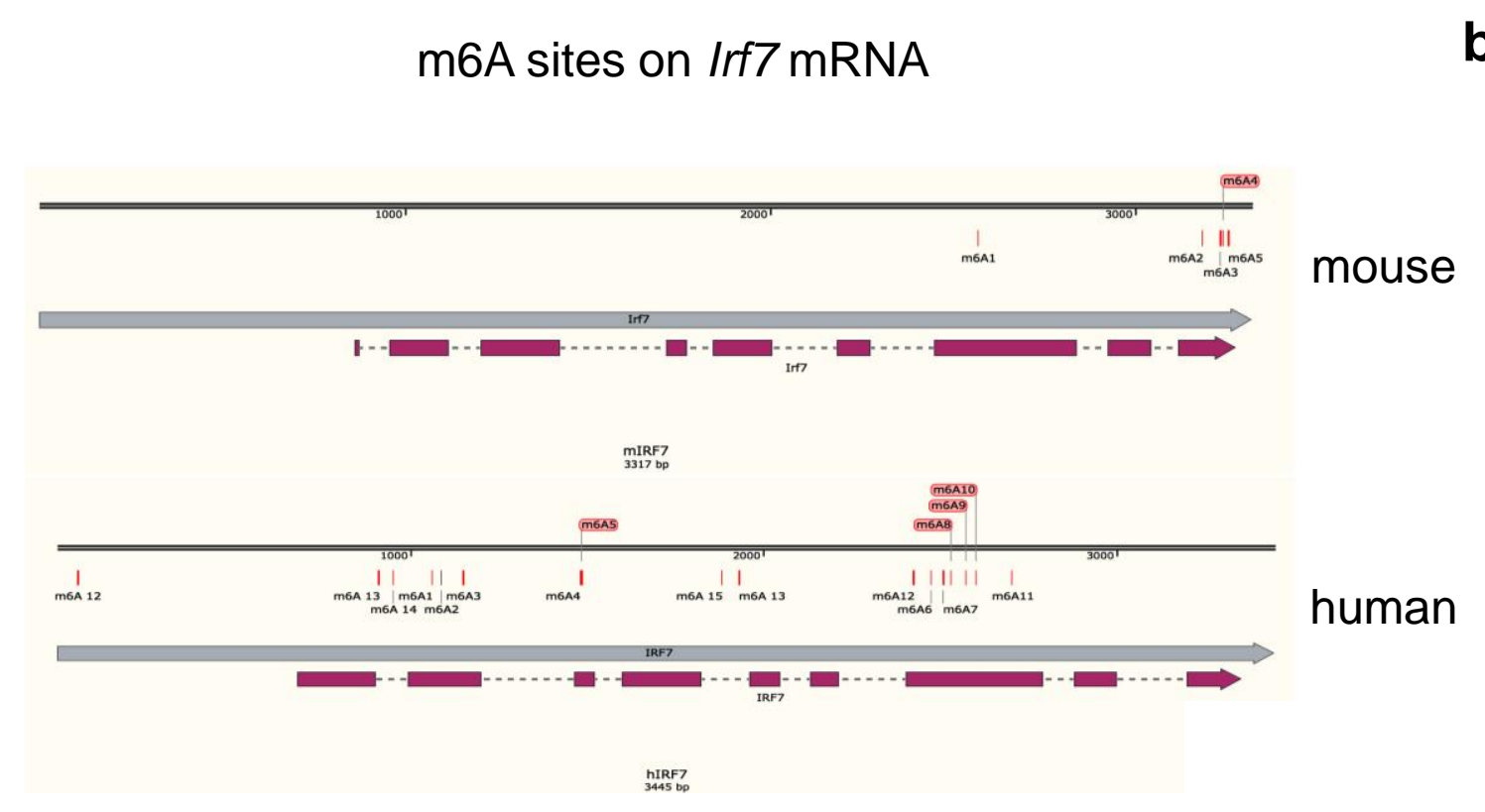

b

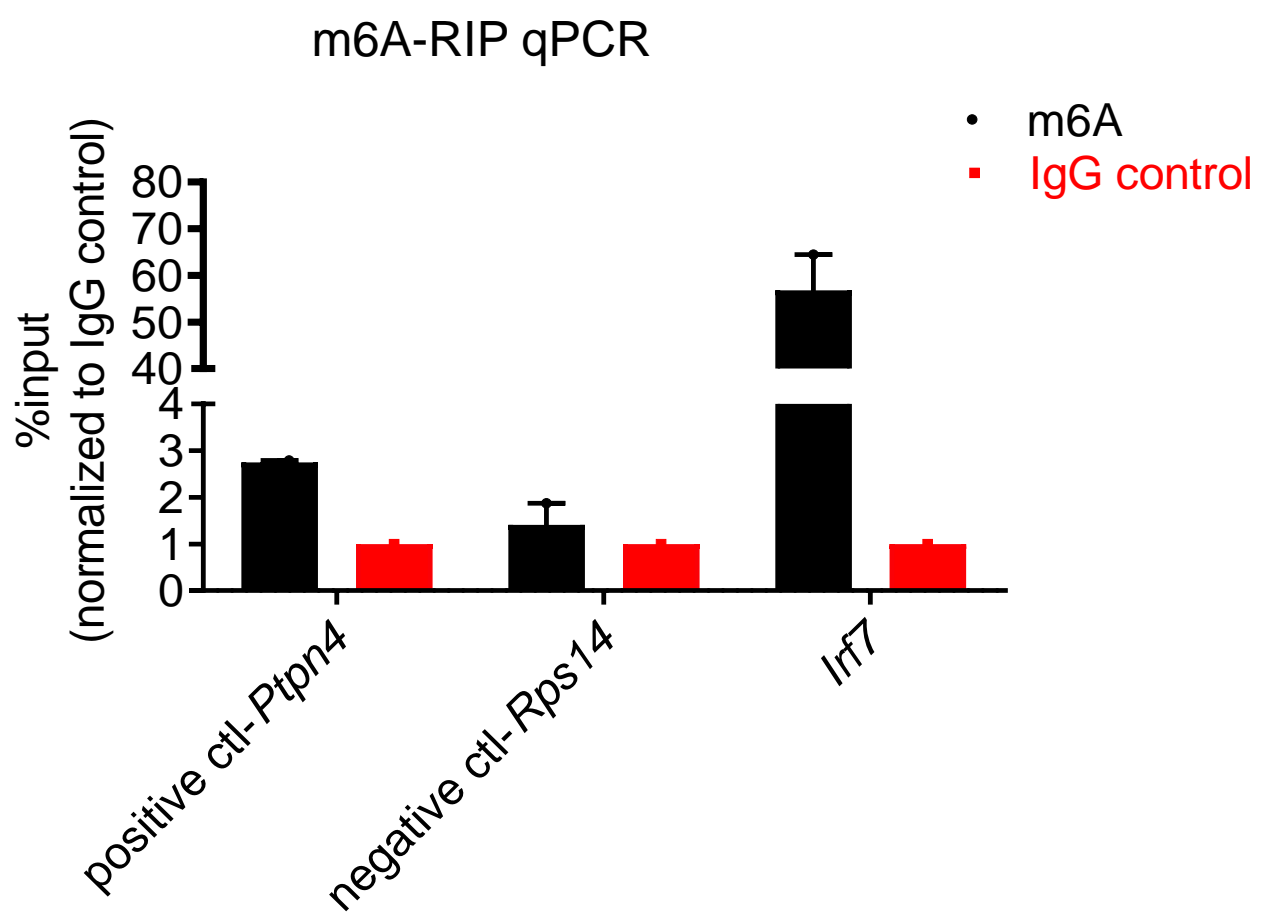

Figure s2. Characterization of m6A modifications on Irf7 mRNA.

(a) Predicted m6A sites of mouse or human IRF7 on the genomes.

(b) m6A-RIP-qPCR confirms Irf7 as an m6A-modified gene in IECs. Mice were infected by RV for 2 days. Fragmented RNA was incubated with an anti-m6A antibody (Sigma Aldrich ABE572) or IgG IP Grade Rabbit polyclonal antibody(abcam, lot: 934197). The eluted RNA and input were processed as described in 'RT-qPCR' section, the data

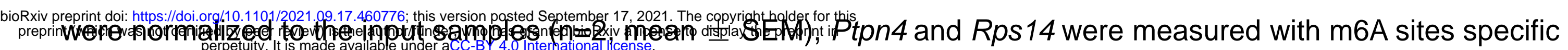
qPCR primer as positive control and negative control, Irf7 was measured with predicted m6A sites specific qPCR primer. 

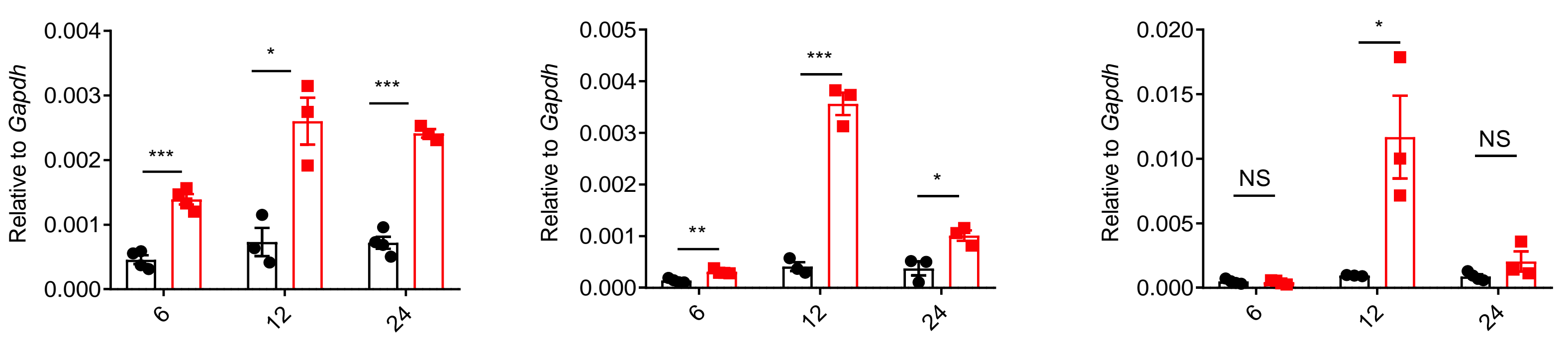

IFIT1
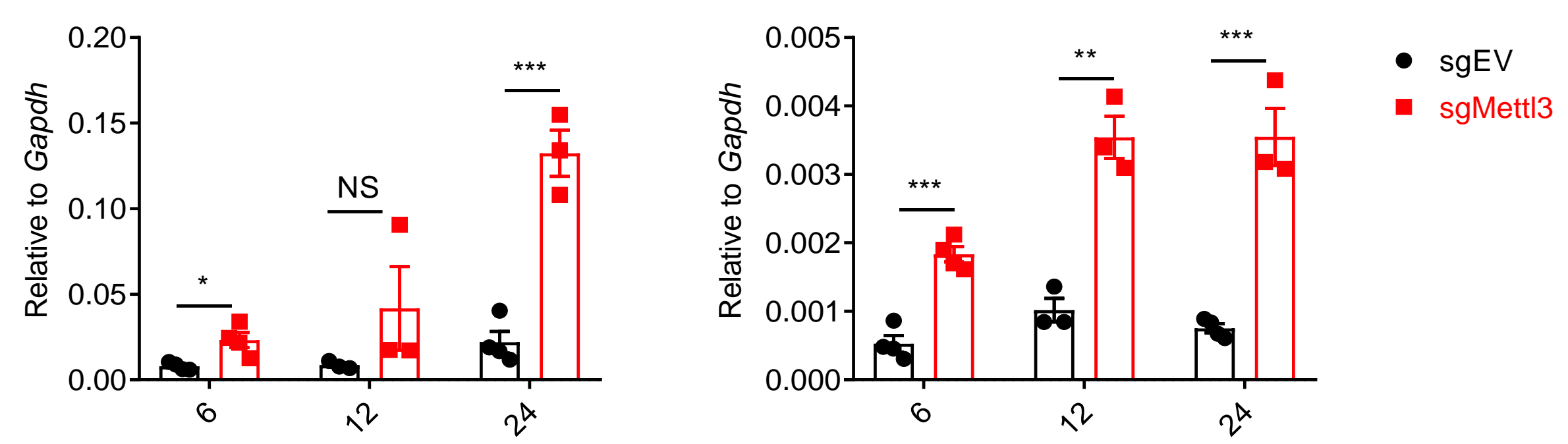

bioRxiv preprint doi: https:///dio.org/10.1101/2021.09.17.460776; this version posted September 17, 2021. The copyright holder for this
preprint (which was not certified by peer review) is the authorffunder, who has granted bioRxiv a license to display the preprint in

b

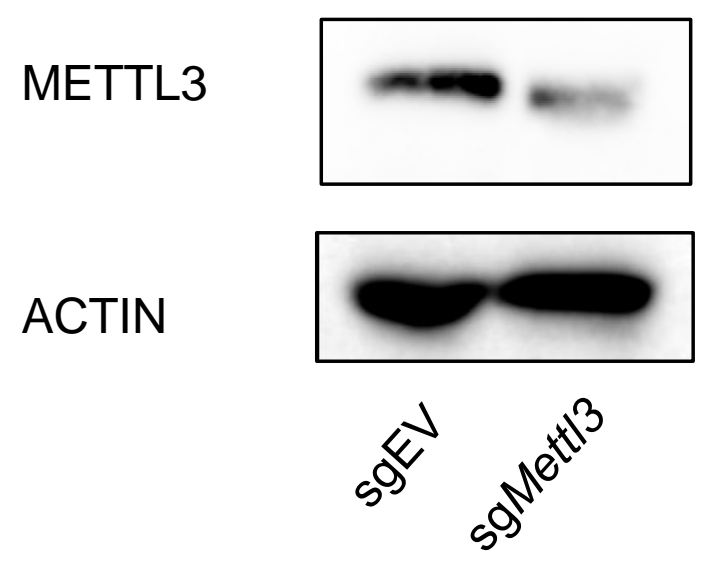

Figure s3. METTL3 knockdown in HT-29 cells results in increased IFN response.

(a) qPCR analysis of IFN/ISGs in Rhesus rotavirus-infected Mett/3 WT and KD HT-29 cells at indicated hours post infection (hpi) (mean \pm SEM), statistical significance was determined by Student's t-test $\left({ }^{*} P<0.05,{ }^{* *} P<0.005,{ }^{* * *} P<0.001\right.$, NS., not significant), at least three replicate experiments were performed.

(b) Knock down efficiency of METTL3 in HT-29 cells. 


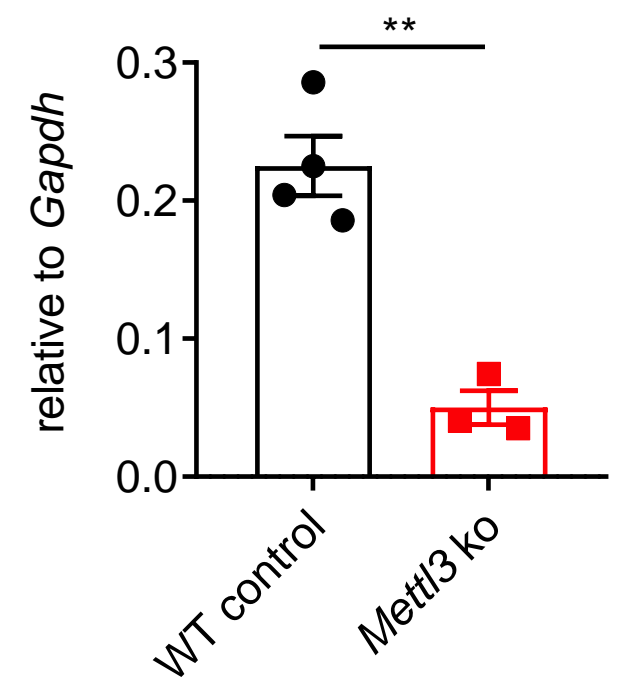

IRF7 in MA104

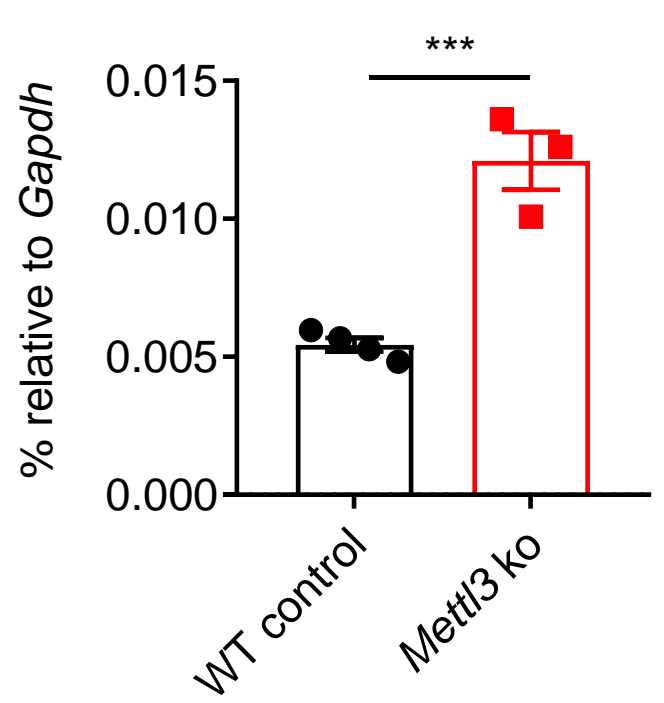

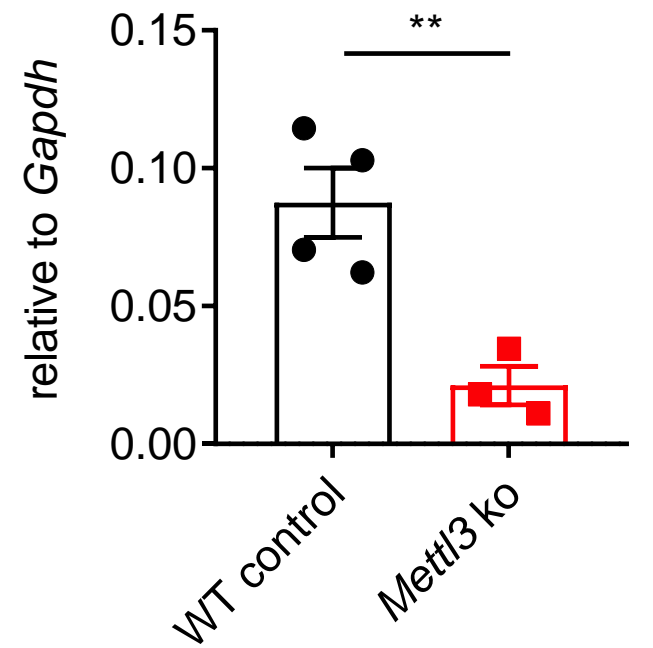

IFIT1 in MA104

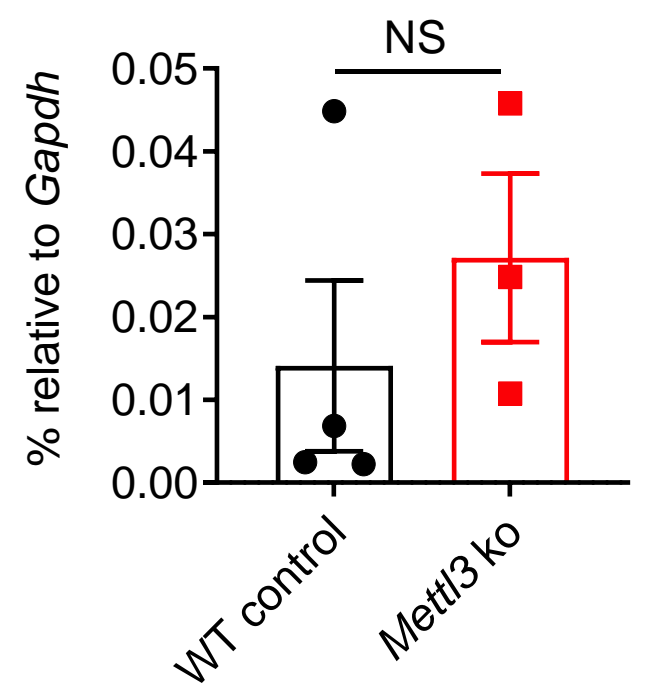

IFNB in MA104

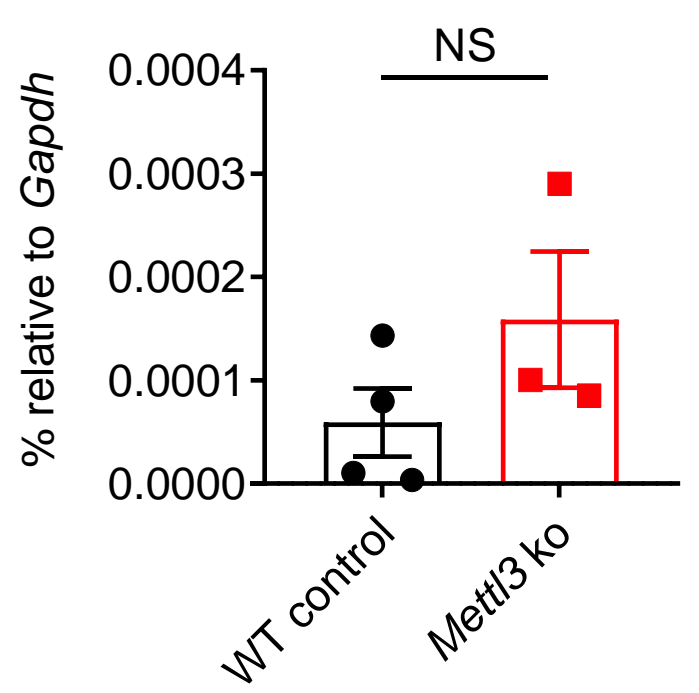

IFNL in MA104

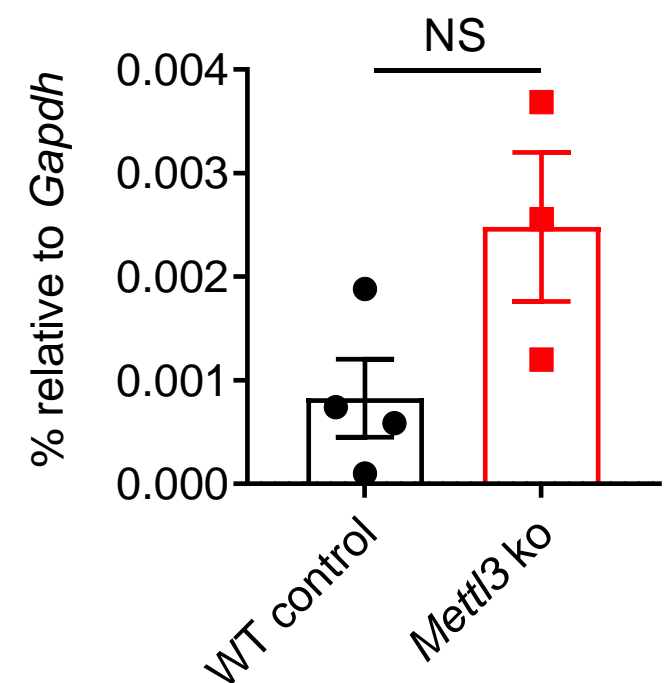

bioRxiv preprint doi: https://doi.org/10.1101/2021.09.17.460776; this version posted September 17, 2021. The copyright holder for this

wh

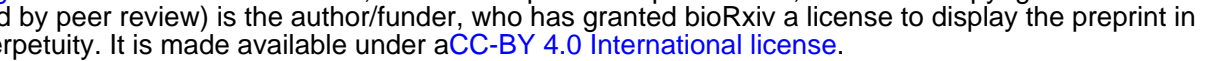

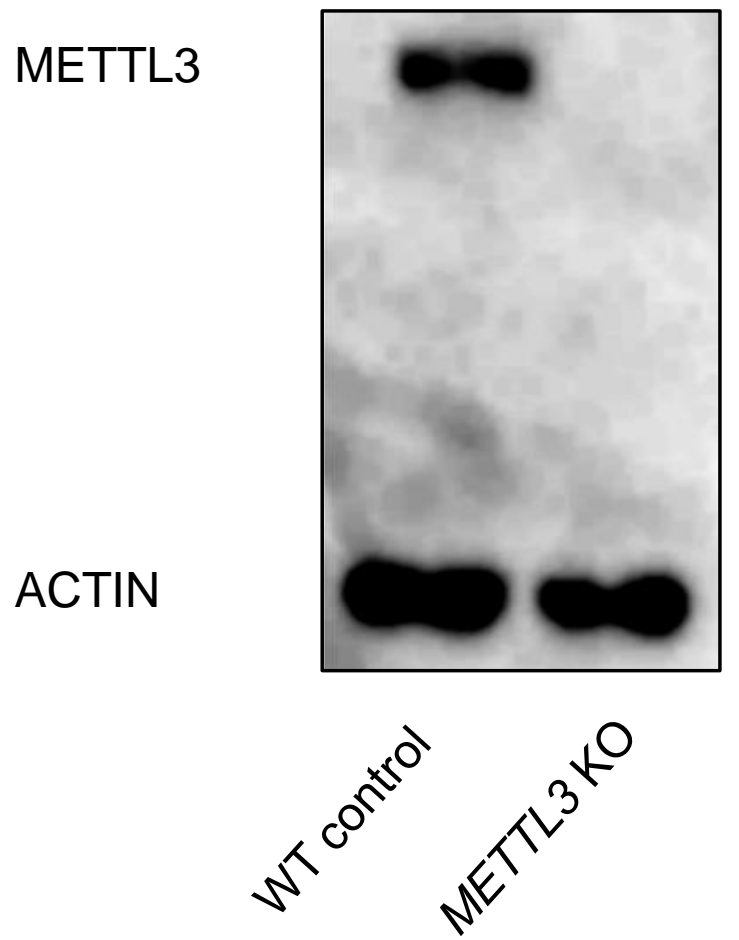

Figure s4. METTL3 deficiency in MA104 cells results in increased resistance to Rhesus rotavirus infection

(a) QPCR analysis of viral RNAs, IFNs, and ISGs in Rhesus rotavirus-infected Mett/3 WT and KO MA104 cells at 24 hours post infection (hpi) (WT control $n=4$, Mett/3 ko $n=3$, mean $\pm S E M)$, statistical significance was determined by Student's t-test $\left({ }^{*} P<0.05\right.$, ${ }^{* *} \mathrm{P}<0.005,{ }^{* * *} \mathrm{P}<0.001, \mathrm{NS} .$, not significant)

(b) Knock out efficiency of METTL3 in MA104 cells. 
a

Irf7 in ileum

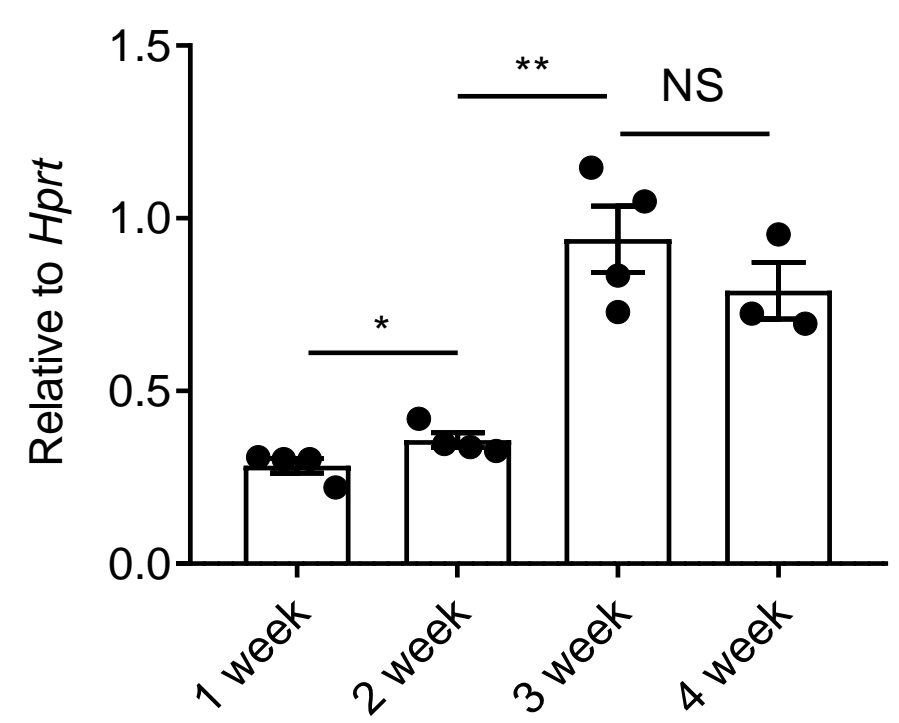

Isg15 in ileum

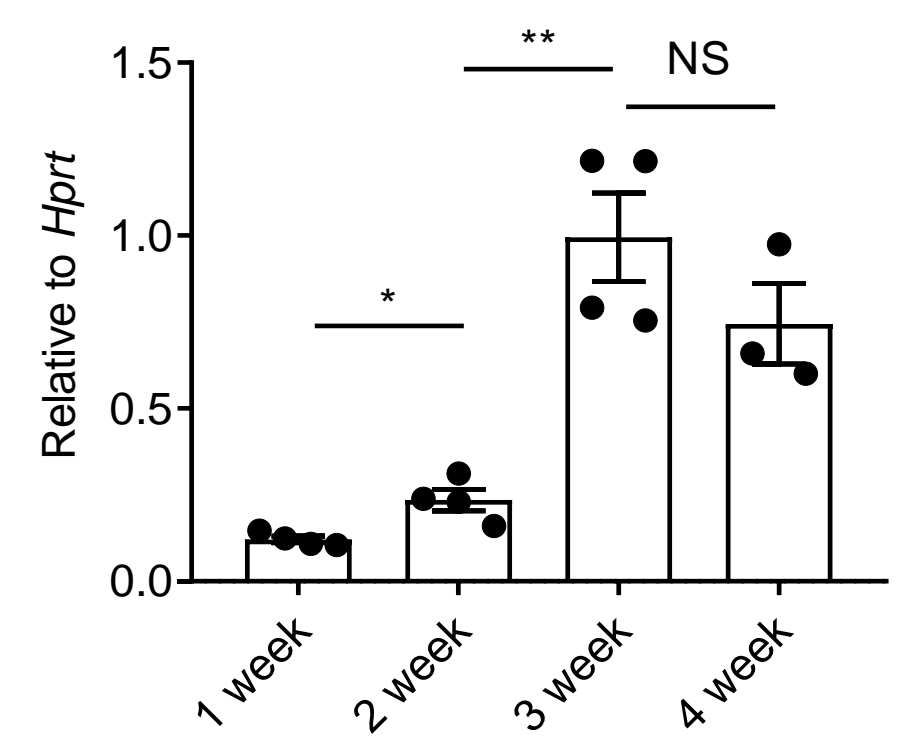

Oas $1 b$ in ileum

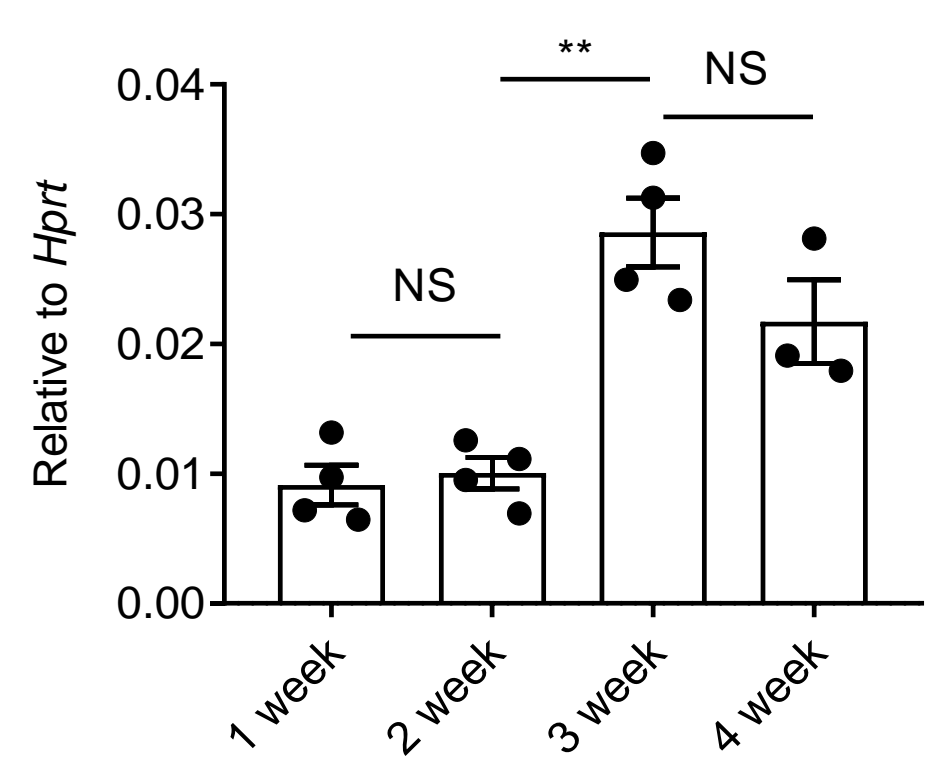

Figure s5. Expression of Irf7 and ISGs in ileum from mice at the ages of 1-4 weeks post birth

(a) qPCR analysis of indicated genes in the ileum ( 1 week $n=4,2$ week $n=4$, 3 week $n=4$, 4 week $n=3$, mean \pm SEM).

Statistical significance was determined by Student's t-tests between ages $\left({ }^{*} P<0.05,{ }^{* *} P<0.005\right.$, NS., not significant). 
a

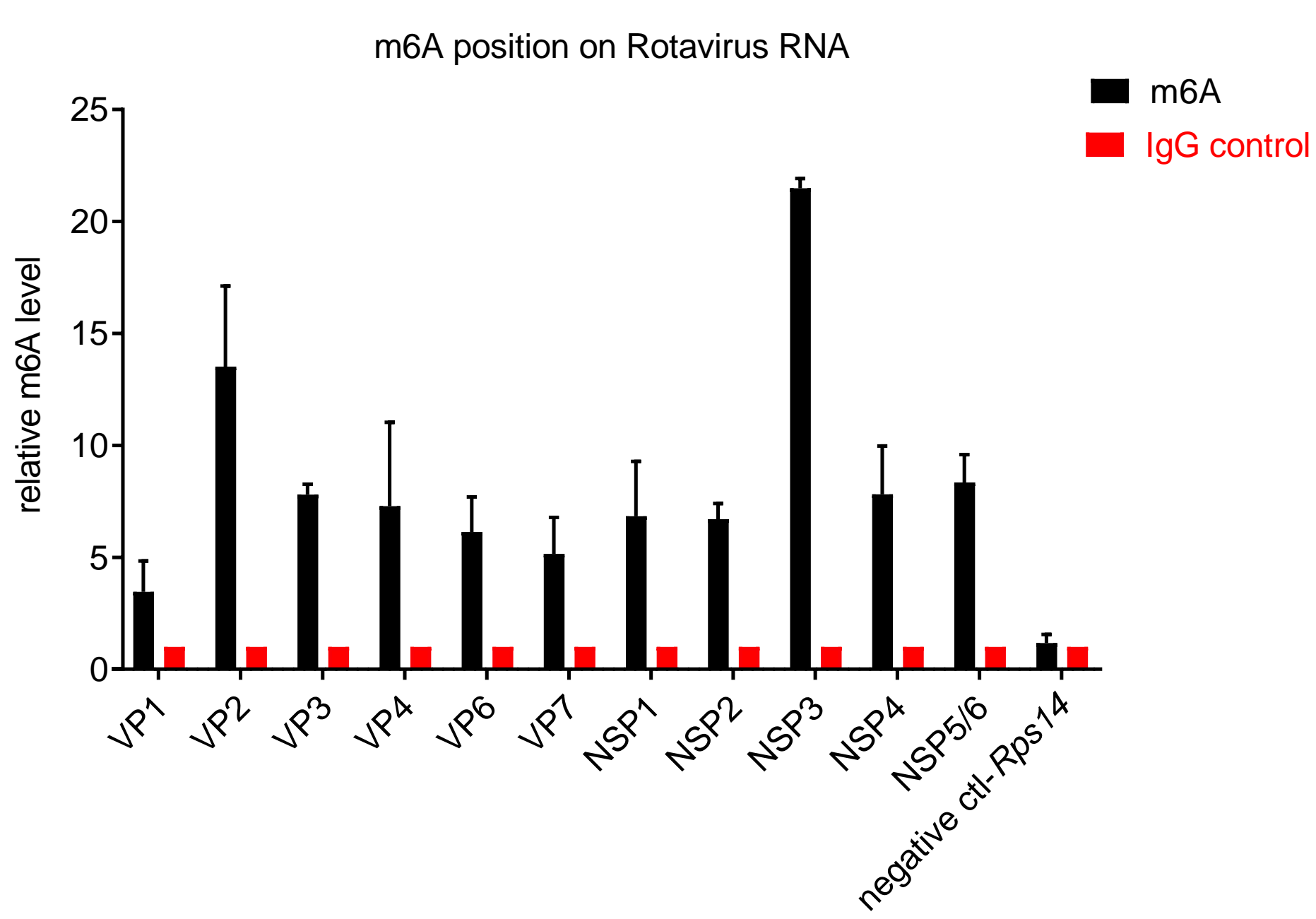

Figure s6. m6A-RIP-qPCR analysis of the predicted m6A sites on Rotavirus RNA.

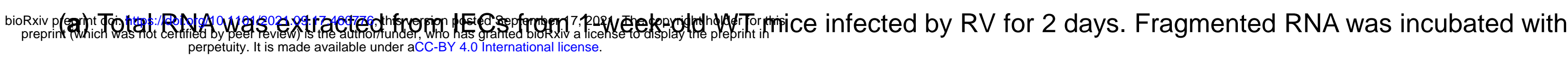
an anti-m6A antibody (Sigma Aldrich ABE572) and IgG IP Grade Rabbit polyclonal antibody(abcam, lot: 934197). The eluted RNA and input were processed as described in 'RT-qPCR' section, the data were normalized to the input samples ( $n=2$, mean \pm SEM), Rps14 was chosen as a negative control. 
a
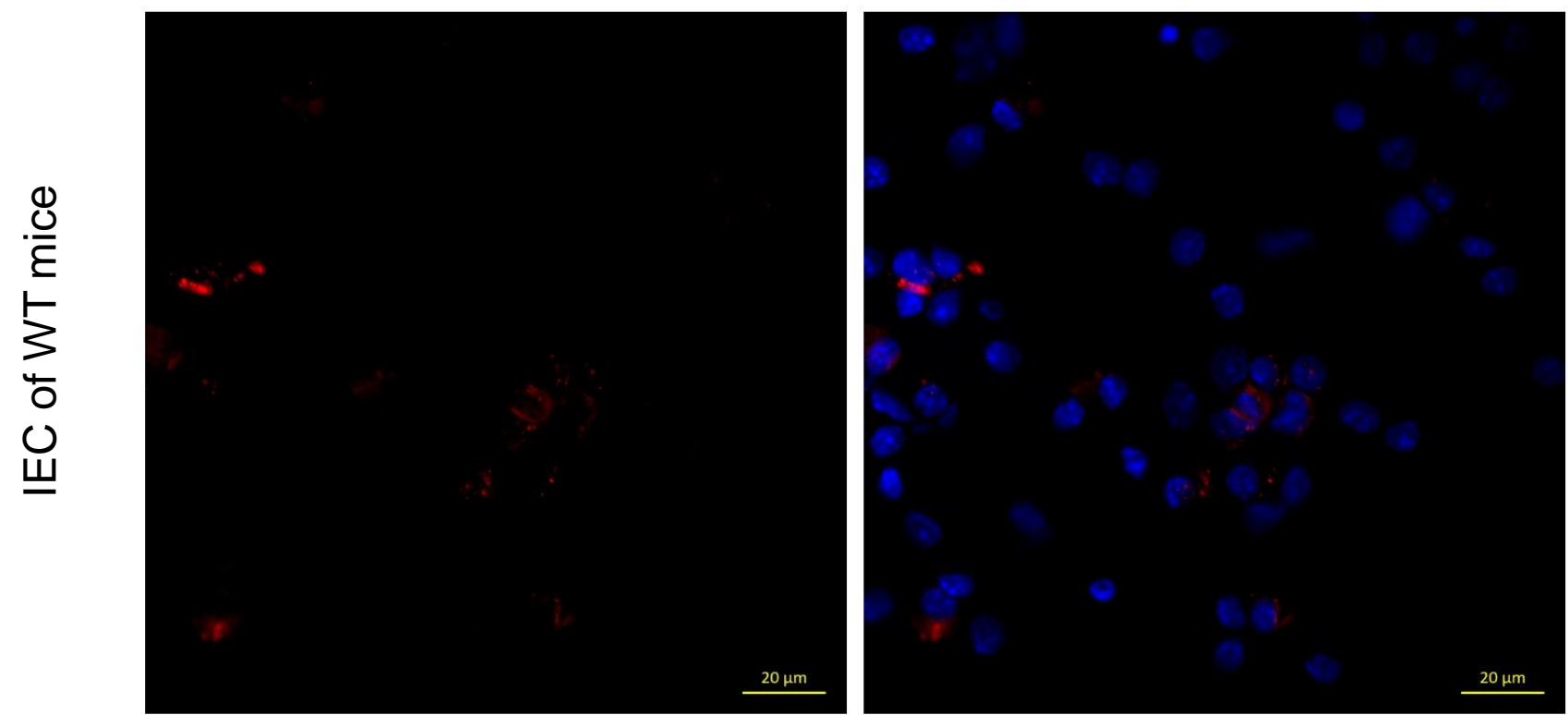

J2

DAPI
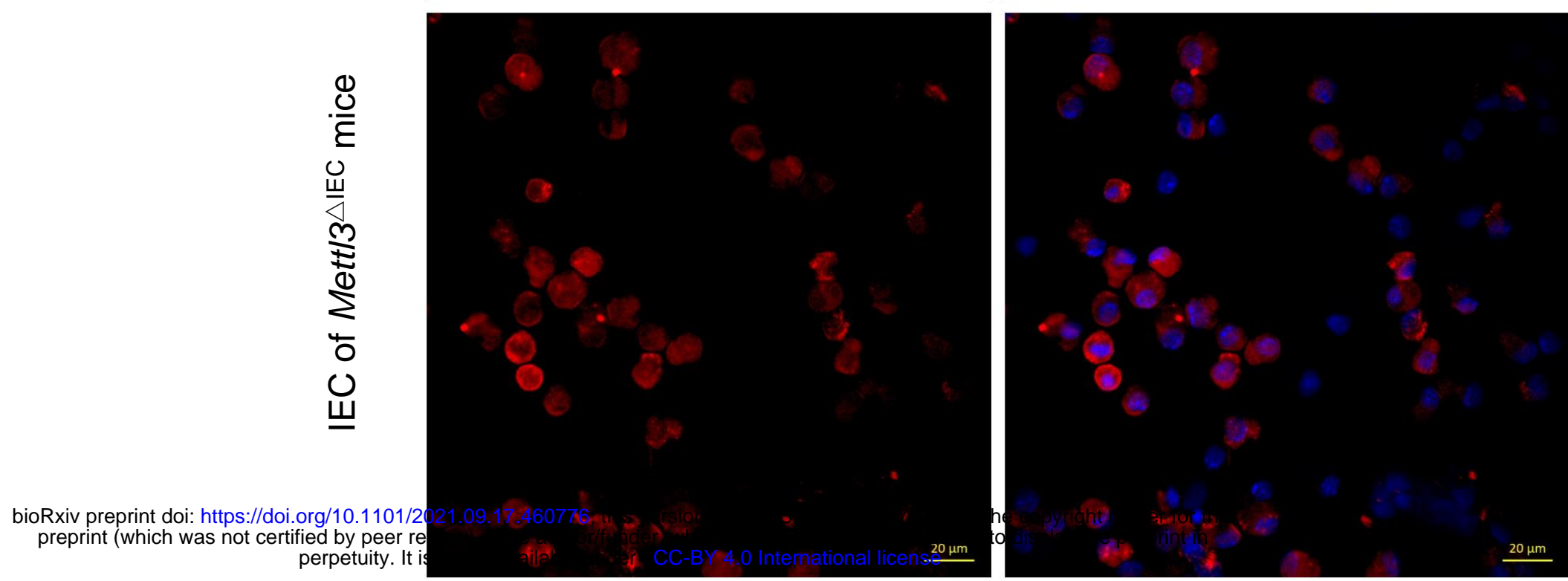

Figure s7. Mett/3 deficiency leads to aberrant dsRNA formation in isolated IECs.

(a) IECs from 6-weeks-old Mett/3 $3^{\Delta E C}$ mice as well as the WT littermate controls were isolated. Double-stranded RNA (dsRNA) was labeled by immunostaining with a mouse monoclonal antibody J2 (Scisons), cells nuclei were visualized with 4,6-diamidino-2phenylindole (DAPI, Invitrogen). All fluorescence images were analyzed via confocal imaging using Zeiss LSM880. 